\title{
The concordance invariant tau in link grid homology
}

\author{
Alberto CAVAllo
}

\begin{abstract}
We introduce a generalization of the Ozsváth-Szabó $\tau$-invariant to links by studying a filtered version of link grid homology. We prove that this invariant remains unchanged under strong concordance and we show that it produces a lower bound for the slice genus of a link. We show that this bound is sharp for torus links and we also give an application to Legendrian link invariants in the standard contact 3 -sphere.
\end{abstract}

$57 \mathrm{M} 25,57 \mathrm{M} 27$

\section{Introduction}

Link Floer homology is an invariant for knots and links in three-manifolds, discovered in 2003 by Ozsváth and Szabó [8] and independently by Jacob Rasmussen [12], in his PhD thesis. It is the homology of a chain complex whose generators are combinatorially defined, and whose differential counts pseudo-holomorphic disks. Grid diagrams are simple combinatorial presentation of links in $S^{3}$, dating back to the $19^{\text {th }}$ century. A grid diagram is an $l \times l$ grid of squares, $l$ of which are marked with an $O$ and $l$ of which are marked with an $X$. A projection of a link together with an orientation on it can be associated to a grid diagram $D$. These grids can be used to give a simpler reformulation of link Floer homology, called grid homology. Of course these two homologies are isomorphic, nevertheless grid homology can be easier to study.

In this paper we use the same notation of the book "Grid homology for knots and links" [7]. In this book particular attention is given to two versions of the grid homology of a link $L$ : the simply blocked grid homology $\widehat{G H}(L)$ and the collapsed unblocked grid homology $c G H^{-}(L)$; both these homology groups are invariant under link equivalence. We study a slightly different version of $\widehat{G H}(L)$. Let us denote with $\mathbb{F}$ the field with two elements; we start constructing a filtered $\mathbb{F}$-complex $(\widehat{G C}(D), \widehat{\partial})$ from a grid diagram $D$, equipped with an increasing $\mathbb{Z}$-filtration $\mathcal{F}$ and we prove that $\mathcal{F}$ induces a filtration in homology, leading to the filtered homology group $\widehat{\mathcal{G H}}(L)$. The latter is not completely unrelated to $\widehat{G H}(L)$ as we see in Section 2 .

We can extract a numerical invariant from the homology $\widehat{\mathcal{G H}}(L)$, the integer-valued function $T_{L}$ : $\mathbb{Z} \times \mathbb{Z} \longrightarrow \mathbb{Z}_{\geqslant 0}$, with the following properties.

Proposition 1.1 i) The function $T_{L}$ is supported in $\{1-n, \ldots, 0\} \times \mathbb{Z}$ and $\sum_{d, s \in \mathbb{Z}} T_{L}(d, s)=2^{n-1}$, where $n$ is the number of components of $L$.

ii) If $L^{*}$ is the mirror of the $n$-component link $L$ then

$$
T_{L^{*}}(d, s)=T_{L}(-d+1-n,-s) \quad \text { for any } d, s \in \mathbb{Z} .
$$

iii) If $L_{1} \# L_{2}$ is a connected sum of $L_{1}$ and $L_{2}$ then $T_{L_{1} \# L_{2}}$ is the convolution product of $T_{L_{1}}$ and $T_{L_{2}}$. 
iv) If $L$ is a quasi-alternating link then $T_{L}$ is determined by the signature of $L$.

Moreover, in Section 4 we prove the following theorem, which is similar to what Pardon proved in [10] for Lee homology. We say that a strong cobordism is a cobordism $\Sigma$, between two links $L_{1}$ and $L_{2}$, such that every connected component of $\Sigma$ is a knot cobordism between a component of $L_{1}$ and one of $L_{2}$; in particular $L_{1}$ and $L_{2}$ have the same number of components. If $g(\Sigma)=0$ then $\Sigma$ is a strong concordance.

Theorem 1.2 The function $T$ is a strong concordance invariant. In other words, if $L_{1}$ and $L_{2}$ are strongly concordant then $T_{L_{1}}(d, s)=T_{L_{2}}(d, s)$ for every $d, s \in \mathbb{Z}$.

In Section 3 we show that $T_{L}(0, s)$ is non-zero only for one value of $s$. We call this integer $\tau(L)$, and, as the name suggests, it coincides with the classical $\tau$ for knots defined in [8]. More precisely, we prove the following statement.

Theorem 1.3 For an n-component link the $\tau$-set, defined in [7] as -1 times the Alexander gradings of a homogeneous, free generating set of the torsion-free quotient of $c \mathrm{GH}^{-}(L)$ as an $\mathbb{F}[U]$-module, coincides with the $2^{n-1}$ (with multiplicity) values of $s$ where the function $T$ is supported.

For a knot $K$, where the $\tau$-set is just $\tau(K)$, we have that $T_{K}(d, s)$ is non-zero only for $(d, s)=$ $(0, \tau(K))$.

From Theorem 1.2 we know that $\tau(L)$ is a strong concordance invariant. Furthermore, it gives a lower bound for the slice genus $g_{4}(L)$, that is the minimum genus of a compact, oriented, smoothly and properly embedded surface in $D^{4}$ with $L$ as boundary.

Proposition 1.4 For every $n$-component link $L$ we have

$$
|\tau(L)|+1-n \leqslant g_{4}(L) .
$$

We use this lower bound to give another proof that, for the positive torus link $T_{q, p}$, we have

$$
g_{4}\left(T_{q, p}\right)=\frac{(p-1)(q-1)+1-\operatorname{gcd}(q, p)}{2} \text { for any } q \leqslant p .
$$

This was already proved by the author in [1] using the Rasmussen $s$-invariant.

Finally, we use $\tau(L)$ to prove a generalization of the Thurston-Bennequin number upper bound, given by Olga Plamenevskaya in [11], to $n$-component Legendrian links. A brief introduction on Legendrian knots and links can be found in [2].

Proposition 1.5 Consider a Legendrian $n$-component link $\mathcal{L}$ of link type $L$ in $S^{3}$ equipped with the standard contact structure. Then the following inequality holds:

$$
\operatorname{tb}(\mathcal{L})+|\operatorname{rot}(\mathcal{L})| \leqslant 2 \tau(L)-n .
$$

Equation (3) gives a lower bound for $\tau$ and, using Equation (1), also the following lower bound for the slice genus of $L$ :

$$
\operatorname{tb}(\mathcal{L})+|\operatorname{rot}(\mathcal{L})| \leqslant 2 g_{4}(L)+n-2,
$$

generalizing a result of Rudolph [13] for knots. In Section 6 we give an example where this bound is sharp. Moreover, Equation (3) can also give an upper bound for $\mathrm{TB}(L)$, the maximal Thurston-Bennequin number of a link $L$. 
Proposition 1.6 For every $n$-component link $L$ we have

$$
T B(L) \leqslant 2 \tau(L)-n .
$$

In particular for a quasi-alternating link, since from iv) in Proposition 1.1 the invariant $\tau$ is determined by the signature, we have the following result that Plamenevskaya proved for alternating knots in [11].

Corollary 1.7 If $L$ is a quasi-alternating link then we have that

$$
T B(L) \leqslant-1-\sigma(L) .
$$

As we show in Section 6, the upper bound in Corollary 1.7 gives the following proposition.

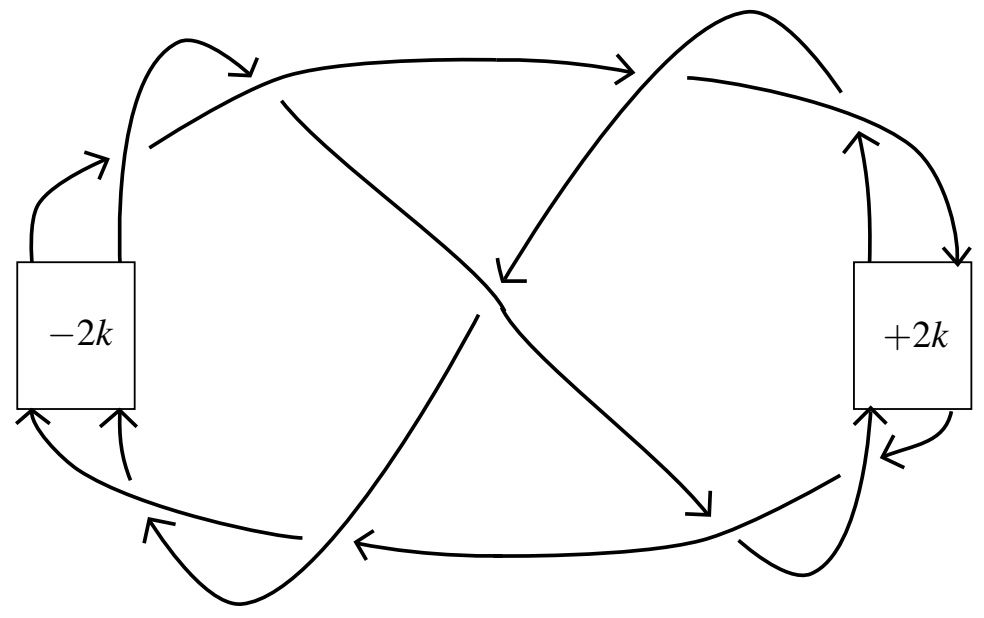

Figure 1: A diagram of $L^{k}$. For $k=0$ we have the link $L 9_{40}^{a}$.

Proposition 1.8 The links $L^{k}$ in Figure 1 are a family of two component links, whose components $L_{i}^{k}$ are unknots with linking number zero, such that $T B\left(L^{k}\right)$ is arbitrarily small.

The paper is organized as follows. In Section 2 we define the filtered chain complex $\widehat{G C}(D)$ and the homology group $\widehat{\mathcal{G H}}(L)$. In Section 3 we introduce the function $T_{L}$ and we prove Proposition 1.1. In Section 4 we construct maps in homology, induced by a cobordism $\Sigma$ between two links $L_{1}$ and $L_{2}$ and we use them to prove Theorem 1.2 and Proposition 1.4. In Section 5 we talk briefly about the filtered version of $c G H^{-}(L)$ and we explain the proof of Theorem 1.3. Finally, in Section 6 we give some applications, including the proof of Equations (2) and (3).

\section{Acknowledgements}

The author would like to thank András Stipsicz for the many helpful conversations and the lots of time spent meeting and discussing mathematics. The author is supported by the ERC Grant LDTBud from the Alfréd Rényi Institute of Mathematics and a Full Tuition Waiver for a Doctoral program at Central European University. 


\section{Filtered simply blocked link grid homology}

\subsection{The complex}

We always suppose that a link is oriented. We denote by $D$ a toroidal grid diagram that represents an $n$-component link $L$. The number $\operatorname{grd}(D)$ is the number of rows and columns in the grid. The

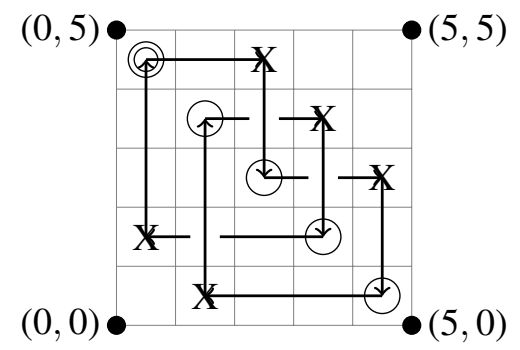

Figure 2: A grid diagram of the positive trefoil knot.

orientation in the diagram is taken by going from the $X$ to the $O$-markings in the columns and the opposite in the rows. Vertical lines are numbered from left to right and horizontal lines from bottom to top, as shown in Figure 2. We identify the boundaries of the grid in order to make it a fundamental domain of a torus; then the lines of the diagram are embedded circles in this torus. Any $\operatorname{grd}(D)$-tuple of points $x$ in the grid, with the property that each horizontal and vertical circle contains exactly one of the elements of $x$, is called a grid state of $D$.

Consider the set of the $O$-markings $\mathbb{O}=\left\{O_{1}, \ldots, O_{\operatorname{grd}(D)}\right\}$. We call special $O$-markings a non-empty subset $s \mathbb{O} \subset \mathbb{O}$ that contains at most one $O$-marking from each component of $L$, while we call the others normal $O$-markings. We represent the special ones with a double circle in the grid diagram. In the paper we usually consider only the case when $\#|s \mathbb{O}|=n$, which means there is exactly one special $O$-marking on each component. We talk about the general case in Subsection 3.2. From now on there are always $n$ special $O$-markings in a grid diagram, unless it is explicitely written differentely.

We define the simply blocked complex $\widehat{G C}(D)$ as the free $\mathbb{F}\left[V_{1}, \ldots, V_{\operatorname{grd}(D)-n}\right]$-module, where $\mathbb{F}=\mathbb{Z} / 2 \mathbb{Z}$, over the grid states $S(D)=\left\{x_{1}, \ldots, x_{\operatorname{grd}(D) !}\right\}$.

We associate to every grid state $x$ the integer $M(x)$, called the Maslov grading of $x$, defined as follows:

$$
M(x)=M_{\mathbb{O}}(x)=\mathcal{J}(x-\mathbb{O}, x-\mathbb{O})+1 ;
$$

where $\mathcal{J}(P, Q)=\sum_{a \in P} \#\{(a, b) \in(P, Q) \mid b$ has both coordinates strictly bigger than the ones of $a\}$ with coordinates taken in the interval $[0, \operatorname{grd}(D))$.

Then we have the Maslov $\mathbb{F}$-splitting

$$
\widehat{G C}(D)=\bigoplus_{d \in \mathbb{Z}} \widehat{G C}_{d}(D)
$$


where $\widehat{G C}_{d}(D)$ is the finite dimensional $\mathbb{F}$-vector space generated by the elements $V_{1}^{l_{1}} \cdot \ldots \cdot V_{m}^{l_{m}} x$, with $x \in S(D)$ and $m=\operatorname{grd}(D)-n$, such that

$$
M\left(V_{1}^{l_{1}} \cdot \ldots \cdot V_{m}^{l_{m}} x\right)=M(x)-2 \sum_{i=1}^{m} l_{i}=d .
$$

We define another integer-valued function on grid states, the Alexander grading $A(x)$, with the formula

$$
A(x)=\frac{M(x)-M_{\mathbb{X}}(x)}{2}-\frac{\operatorname{grd}(D)-n}{2} ;
$$

where $M_{\mathbb{X}}(x)$ is defined in Equation (5), replacing the set $\mathbb{O}$ with $\mathbb{X}$. For the proof that $A(x)$ is really an integer we refer to Chapter 8 in [7].

Now we introduce an increasing filtration on $\widehat{G C}(D)$ such that

$$
\mathcal{F}^{s} \widehat{G C}(D)=\bigoplus_{d \in \mathbb{Z}} \mathcal{F}^{s} \widehat{G C}_{d}(D)
$$

and where $\mathcal{F}^{s} \widehat{G C}_{d}(D)$ is generated over $\mathbb{F}$ by the elements $V_{1}^{l_{1}} \cdot \ldots \cdot V_{m}^{l_{m}} x$ with Maslov grading $d$ and Alexander grading

$$
A\left(V_{1}^{l_{1}} \cdot \ldots \cdot V_{m}^{l_{m}} x\right)=A(x)-\sum_{i=1}^{m} l_{i} \leqslant s
$$

\subsection{The differential}

First we take $x, y \in S(D)$. The set $\operatorname{Rect}(x, y)$ is defined in the following way: it is always empty except when $x$ and $y$ differs only by a pair of points, say $\{a, b\}$ in $x$ and $\{c, d\}$ in $y$; then $\operatorname{Rect}(x, y)$ consists of the two rectangles in the torus represented by $D$ that have bottom-left and top-right vertices in $\{a, b\}$ and bottom-right and top-left vertices in $\{c, d\}$. We call $\operatorname{Rect}^{\circ}(x, y) \subset \operatorname{Rect}(x, y)$ the subset of the rectangles which do not contain a point of $x$ (or $y$ ) in their interior.

The differential $\widehat{\partial}$ is defined as follows:

$$
\widehat{\partial} x=\sum_{y \in S(D)} \sum_{\substack{r \in \operatorname{Rect}^{\circ}(x, y) \\ r \cap s \mathbb{O}=\emptyset}} V_{1}^{O_{1}(r)} \cdot \ldots \cdot V_{m}^{O_{m}(r)} y \text { for any } x \in S(D)
$$

where $O_{i}(r)=\left\{\begin{array}{ll}1 & \text { if } O_{i} \in r \\ 0 & \text { if } O_{i} \notin r\end{array}\right.$. Here $\left\{O_{1}, \ldots, O_{m}\right\}$ is the set of the $m=\operatorname{grd}(D)-n$ normal $O$-markings.

We extend $\widehat{\partial}$ to $\widehat{G C}_{d}(D)$ linearly, and we call it $\widehat{\partial}_{d}$, then again to the whole $\widehat{G C}(D)$ in the following way: $\widehat{\partial}\left(V_{i} x\right)=V_{i} \cdot \widehat{\partial} x$ for every $i=1, \ldots, m$ and $x \in S(D)$. Since $\widehat{\partial}$ keeps the filtration and drops the Maslov grading by 1 (Lemma 13.2.3 in [7]), we have maps

$$
\widehat{\partial}_{d, s}: \mathcal{F}^{s} \widehat{G C}_{d}(D) \longrightarrow \mathcal{F}^{s} \widehat{G C}_{d-1}(D)
$$

where $\widehat{\partial}_{d, s}$ is the restriction of $\widehat{\partial}_{d}$ to the subspace $\mathcal{F}^{s} \widehat{G C}_{d}(D) \subset \widehat{G C}_{d}(D)$. Furthermore $\widehat{\partial} \circ \widehat{\partial}=0$ (Lemma 13.2.2 in [7]). 


\subsection{The homology}

We define the homology group $\widehat{\mathcal{G H}}_{d}(D)$ as the quotient space $\frac{\operatorname{Ker} \widehat{\partial}_{d}}{\operatorname{Im} \widehat{\partial}_{d+1}}$. Moreover, we introduce the subspaces $\mathcal{F}^{s} \widehat{\mathcal{G H}}_{d}(D)$ as follows: consider the projection $\pi_{d}: \operatorname{Ker} \widehat{\partial}_{d} \rightarrow \widehat{\mathcal{G H}}_{d}(D)$. Since Ker $\widehat{\partial}_{d, s}=\operatorname{Ker} \widehat{\partial}_{d} \cap \mathcal{F}^{s} \widehat{G C}_{d}(D)$ we say that

$$
\mathcal{F}^{s} \widehat{\mathcal{G H}}_{d}(D)=\pi_{d}\left(\operatorname{Ker} \widehat{\partial}_{d, s}\right)
$$

for every $s \in \mathbb{Z}$. Ker $\widehat{\partial}_{d, s} \subset \operatorname{Ker} \widehat{\partial}_{d, s+1}$ implies that the filtration $\mathcal{F}$ descends to homology. We see immediately that each $\mathcal{F}^{s} \widehat{\mathcal{G H}}_{d}(D)$ is a finite dimensional $\mathbb{F}$-vector space.

We can extend the filtration $\mathcal{F}$ on the total homology

$$
\widehat{\mathcal{G H}}(D)=\bigoplus_{d \in \mathbb{Z}} \widehat{\mathcal{G H}}_{d}(D)
$$

by taking

$$
\mathcal{F}^{s} \widehat{\mathcal{G H}}(D)=\bigoplus_{d \in \mathbb{Z}} \mathcal{F}^{s} \widehat{\mathcal{G H}}_{d}(D)
$$

From [7] Chapter 13 we know that the dimension of $\mathcal{F}^{s} \widehat{\mathcal{G H}}_{d}(D)$ as an $\mathbb{F}$-vector space is a link invariant for every $d, s \in \mathbb{Z}$, in particular they are independent of the choice of the special $O$-markings and the ordering of the markings. Hence we can denote them with $\mathcal{F}^{s} \widehat{\mathcal{G H}}_{d}(L)$. Furthermore, [7] Lemma 13.2.5 says that $\left[V_{i} p\right]=[0]$ for every $i=1, \ldots, m$ and $[p] \in \widehat{\mathcal{G H}}(L)$. This means that each homology class can be represented by a combination of grid states and every level $\mathcal{F}^{s} \widehat{\mathcal{G H}}(L)$ is also a finite dimensional $\mathbb{F}$-vector space.

The homology group $\widehat{G H}_{d, s}(L)$ of [7] can be recovered from the complex $\widehat{G C}(D)$ in the following way. We denote the graded object associated to a filtered complex $\mathcal{C}$ the bigraded chain complex $(\operatorname{gr}(\mathcal{C}), \operatorname{gr}(\partial))$, where

$$
\operatorname{gr}(\mathcal{C})_{d, s}=\frac{\mathcal{F}^{s} \mathcal{C}_{d}}{\mathcal{F}^{s-1} \mathcal{C}_{d}}
$$

and $\operatorname{gr}(\partial)$ is the map induced by $\partial$ on $\operatorname{gr}(\mathcal{C})$. Then we have that

$$
\widehat{G H}_{d, s}(L) \cong_{\mathbb{F}} H_{d, s}(\operatorname{gr}(\widehat{G C}(D)), \operatorname{gr}(\widehat{\partial})) .
$$

\section{The invariant tau in the filtered theory}

\subsection{Definitions}

Since $\mathcal{F}^{s-1} \widehat{\mathcal{G H}}_{d}(L) \subset \mathcal{F}^{s} \widehat{\mathcal{G H}}_{d}(L)$, and they are finite dimensional vector spaces, we define the function

$$
T_{L}(d, s)=\operatorname{dim}_{\mathbb{F}} \frac{\mathcal{F}^{s} \widehat{\mathcal{G H}}_{d}(L)}{\mathcal{F}^{s-1} \widehat{\mathcal{G H}}_{d}(L)}
$$

which clearly is still a link invariant.

Our first goal is to see what happens to this function $T$ when we stabilize the link $L$, in other words when we add a disjoint unknot to $L$. Denote the unknot with the symbol $\bigcirc$. We claim that

$$
T_{L \sqcup \bigcirc}(d, s)=T_{L}(d, s)+T_{L}(d+1, s) \quad \text { for any } d, s \in \mathbb{Z} .
$$


Before the proof of Equation (6) it is time for some remarks on filtered chain maps.

Suppose $f:(\mathcal{C}, \partial) \rightarrow\left(\mathcal{C}^{\prime}, \partial^{\prime}\right)$ is a chain map between two filtered chain complexes over $\mathbb{F}$. We say that $f$ is filtered of degree $t$ if $f\left(\mathcal{F}^{s} \mathcal{C}\right) \subset \mathcal{F}^{s+t} \mathcal{C}^{\prime}$ for every $s \in \mathbb{Z}$.

A filtered chain map induces a map in homology that is filtered of the same degree. This means that $f$ induces a map $f_{*}: H_{*}(\mathcal{C}) \rightarrow H_{*}\left(\mathcal{C}^{\prime}\right)$ such that $f_{*}\left(\mathcal{F}^{s} H_{*}(\mathcal{C})\right) \subset \mathcal{F}^{s+t} H_{*}\left(\mathcal{C}^{\prime}\right)$ for any $s \in \mathbb{Z}$.

We say that a linear map $F: H_{*}(\mathcal{C}) \rightarrow H_{*}\left(\mathcal{C}^{\prime}\right)$ is a filtered isomorphism if $F$ is bijective and $F\left(\mathcal{F}^{s} H_{*}(\mathcal{C})\right)=\mathcal{F}^{s} H_{*}\left(\mathcal{C}^{\prime}\right)$ for any $s \in \mathbb{Z}$. We denote with $H_{*}(\mathcal{C}) \cong H_{*}\left(\mathcal{C}^{\prime}\right)$ two filtered isomorphic homology groups such that the isomorphism preserves the grading; more excplicitely this means that $\mathcal{F}^{s} H_{d}(\mathcal{C}) \cong_{\mathbb{F}} \mathcal{F}^{s} H_{d}\left(\mathcal{C}^{\prime}\right)$ for every $d, s \in \mathbb{Z}$.

Moreover, we can associate to a filtered chain map $f: \mathcal{C} \rightarrow \mathcal{C}^{\prime}$ the quotient map

$$
\operatorname{gr}(f): \operatorname{gr}(\mathcal{C}) \longrightarrow \operatorname{gr}\left(\mathcal{C}^{\prime}\right) \text {. }
$$

We call $f$ a filtered quasi-isomorphism if the map $\operatorname{gr}(f)$ induces an isomorphism between $H_{*, *}(\operatorname{gr}(\mathcal{C}))$ and $H_{*, *}\left(\operatorname{gr}\left(\mathcal{C}^{\prime}\right)\right)$ that preserves the gradings. We denote with $\mathcal{C} \cong \mathcal{C}^{\prime}$ two filtered quasi-isomorphic complexes.

From Proposition A.6.1 in [7] we have that if there is a filtered quasi-isomorphism between $(\mathcal{C}, \partial)$ and $\left(\mathcal{C}^{\prime}, \partial^{\prime}\right)$ then $H_{*}(\mathcal{C}) \cong H_{*}\left(\mathcal{C}^{\prime}\right)$. While from Proposition A.8.1 in [7] we know that $\mathcal{C} \cong \mathcal{C}^{\prime}$ if and only if there is a filtered chain homotopy equivalence between $\mathcal{C}$ and $\mathcal{C}^{\prime}$, provided $\mathcal{C}$ and $\mathcal{C}^{\prime}$ are also modules over $\mathbb{F}\left[V_{1}, \ldots, V_{m}\right]$. See [7] Chapter 13 and Appendix A for more details.

Finally, we define the shifted complex $\mathcal{C} \llbracket a, b \rrbracket=\mathcal{C}^{\prime}$ as $\mathcal{F}^{s} \mathcal{C}_{d}^{\prime}=\mathcal{F}^{s-b} \mathcal{C}_{d-a}$. Now, in order to prove Equation (6), we need the following proposition.

Proposition 3.1 For any link $L$ we have $\widehat{\mathcal{G H}}(L \sqcup \bigcirc) \cong \widehat{\mathcal{G H}}(L) \otimes V$, where $V$ is the two dimensional $\mathbb{F}$-vector space with generators in grading and minimal level $(d, s)=(-1,0)$ and $(d, s)=(0,0)$.

Proof Take a grid diagram $D$ for $L$. Then the extended diagram $\bar{D}$, obtained from $D$ by adding one column on the left and one row on the top with a doubly-marked square in the top left, represents the link $L \sqcup \bigcirc$. The circle in the doubly-marked square is forced to be a special $O$-marking and we can also suppose that there is another special $O$-marking just below and right of it, as shown in Figure 3.

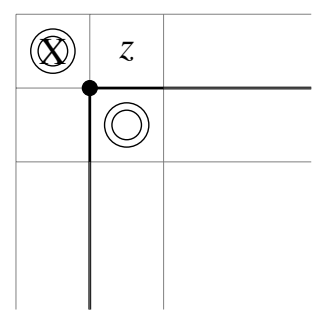

Figure 3: We call the top-left special $O$-marking $O_{0}$.

Let $I(\bar{D})$ denote the set of those generators in $S(\bar{D})$ which have a component at the lower-right corner of the doubly-marked square, and let $N(\bar{D})$ be the complement of $I(\bar{D})$ in $S(\bar{D})$. From the placement of the special $O$-markings, we see that $N(\bar{D})$ spans a subcomplex $\mathbf{N}$ in $\widehat{G C}(\bar{D})$. Moreover, if $\widehat{\partial}_{1}$ is the 
differential in $\widehat{G C}(\bar{D})$ and $\widehat{\partial}_{2}$ the one in $\widehat{G C}(D)$ we can express the restriction of $\widehat{\partial}_{1}$ to the subspace I, spanned by $I(\bar{D})$, with $\widehat{\partial}_{2}+\widehat{\partial}_{\mathbf{N}}$; this because there is a one-to-one correspondence between elements of $I(\bar{D})$ and grid states in $S(D)$. This correspondence induces a filtered quasi-isomorphism $i:\left(\mathbf{I}, \widehat{\partial}_{2}\right) \rightarrow \widehat{G C}(D)$.

Define a map $H: \mathbf{N} \rightarrow \mathbf{I}$ by the formula

$$
H(x)=\sum_{y \in I(\bar{D})} \sum_{\substack{r \in \operatorname{Rect}^{\circ}(x, y) \\ O_{0} \in r}} V_{1}^{O_{1}(r)} \cdot \ldots \cdot V_{m}^{O_{m}(r)} y \quad \text { for any } x \in N(\bar{D}) .
$$

We have that $H$ is a filtered chain homotopy equivalence between $\left(\mathbf{N}, \widehat{\partial}_{1}\right)$ and $\left(\mathbf{I}, \widehat{\partial}_{2}\right)$, which increases the Maslov grading by one, and $H \circ \widehat{\partial}_{\mathbf{N}}=0$. To see the first claim, we mark the square just on the right of $O_{0}$ with $z$ and we define an operator $\widehat{H}_{z}: \mathbf{I} \rightarrow \mathbf{N}$, which counts only rectangles that contain $z$. This operator is a homology inverse of $H$; this and the second claim can be proved in the same way as in Lemma 8.4.7 in [7]. Those two facts together tell us that the following diagram commutes.

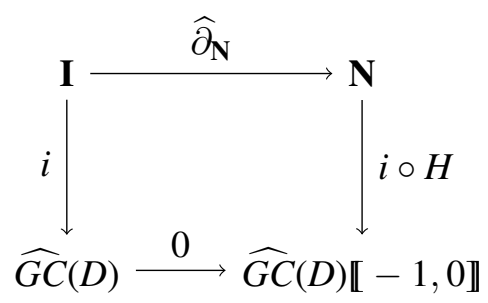

Since $H$ is a filtered chain homotopy equivalence, $i \circ H$ is a filtered quasi-isomorphism, just like the map $i$. Therefore, we can use a filtered version of Lemma A.3.8 in [7] and obtain that the map between the mapping cones

$$
\operatorname{Cone}\left(\widehat{\partial}_{\mathbf{N}}\right)=\widehat{G C}(\bar{D}) \longrightarrow \operatorname{Cone}(0)=\widehat{G C}(D) \otimes V
$$

is a filtered quasi-isomorphism and so the claim follows easily. See also the proof of Lemma 8.4.7 in [7] for other details.

Equation (6) is obtained immediately from Proposition 3.1, in fact we have proved that $\mathcal{F}^{s} \widehat{\mathcal{G H}}_{d}(L \sqcup$ $\bigcirc) \cong \mathcal{F}^{s} \widehat{\mathcal{G H}}_{d}(L) \oplus \mathcal{F}^{s} \widehat{\mathcal{G H}}_{d+1}(L)$ for every $d, s \in \mathbb{Z}$ and so it is enough to apply the definition of $T$.

Now we are able to do some computations. The homology of the unknot can be easily computed by taking the grid diagram of dimension 1, where the square is marked with both $X$ and $O$. The complex has one element of Maslov and Alexander grading 0; then $T_{\bigcirc}(d, s)=1$ if $(d, s)=(0,0)$ and it is 0 otherwise.

Using Equation (6) we get the function $T$ of the $n$-component unlink $\bigcirc_{n}$ :

$$
T_{\bigcirc_{n}}(d, s)=\left\{\begin{array}{cc}
\left(\begin{array}{c}
n-1 \\
k
\end{array}\right) & \text { if }(d, s)=(-k, 0), \quad 0 \leqslant k \leqslant n-1 \\
0 & \text { otherwise }
\end{array} .\right.
$$

We can also see this directly from Proposition 3.1, in fact we have that $\widehat{\mathcal{G H}}\left(\bigcirc_{n}\right) \cong V^{\otimes(n-1)}$.

Now let us consider a grid diagram $D$ of a link $L$. The Maslov grading of the elements of $S(D)$ and the differential $\widehat{\partial}$ are independent of the position of the $X$ 's, once we have fixed the 
special $O$-markings. Since we can always change the $X$-markings to obtain $\bigcirc_{n}$, this means that $\operatorname{dim}_{\mathbb{F}} \widehat{\mathcal{G H}}_{d}(L)=\operatorname{dim}_{\mathbb{F}} \widehat{\mathcal{G H}}_{d}\left(\bigcirc_{n}\right)$ for every $d \in \mathbb{Z}$ and the generators are the same. In particular

$$
\left.\widehat{\mathcal{G H}}(L) \cong_{\mathbb{F}} \widehat{\mathcal{G H}}\left(\bigcirc_{n}\right) \cong_{\mathbb{F}} \mathbb{F}^{2^{n-1}} \quad \text { and } \quad \widehat{\mathcal{G H}}_{d}(L) \cong_{\mathbb{F}} \mathbb{F}^{(n-1}-d\right) \quad \text { when } 1-n \leqslant d \leqslant 0 .
$$

From this we have that $\widehat{\mathcal{G H}}_{0}(L)$ has always dimension 1 and then we define $\tau(L)$ as the only integer $s$ such that $T_{L}(0, s)>0$, as we previously said in the Introduction. We remark that for a knot this version of $\tau$ coincides with the one of Ozsváth and Szabó. See the proof of Theorem 1.3 in Section 5. We also observe that Equation (6) tells that $\tau(L \sqcup \bigcirc)=\tau(L)$.

\subsection{Dropping the special $O$-markings}

In this subsection we study what happens to the homology of the filtered chain complex $(\widehat{G C}(D), \widehat{\partial})$ if the grid diagram $D$ has less than $n$ special $O$-markings.

Let us consider $D$ a grid diagram for an $n$-component link $L$. The set $s \mathbb{O} \subset \mathbb{O}$ contains at most one $O$-marking from each component of $L$, but we have that $\#|s \mathbb{O}| \geqslant 1$. Denote with $m=\operatorname{grd}(D)-\#|s \mathbb{O}|$ the number of normal $O$-markings in $D$. Then we can define our chain complex exactly in the same way as in Section 2; on the other hand, the homology $\widehat{\mathcal{G H}}(D)$ is no longer a link invariant, in fact it clearly depends of the choice of the special $O$-markings.

Nonetheless, we can show that the $\mathbb{F}$-vector space $\widehat{\mathcal{G H}}(D)$ is still finite dimensional. Note that this is not true if instead we consider the simply blocked homology group $\widehat{G H}(D)$.

Proposition 3.2 The homology group $\widehat{\mathcal{G H}}(D)$, defined as in Subsection 2.3, is $\mathbb{F}$-isomorphic to $\widehat{\mathcal{G H}}\left(\bigcirc_{\#|s \mathbb{O}|}\right)$, the homology of the unlink with $\#|s \mathbb{O}|$ components each containing a special $O$-marking. In particular, we have that

$$
\left.\widehat{\mathcal{G H}}(D) \cong_{\mathbb{F}} \mathbb{F}^{2^{\#|s \mathbb{Q}|}-1} \quad \text { and } \quad \widehat{\mathcal{G H}}_{d}(D) \cong_{\mathbb{F}} \mathbb{F}^{(\#|s \mathbb{O}|-1}\right)
$$

when $1-\#|s \mathbb{O}| \leqslant d \leqslant 0$.

Proof As we noted before, the group $\widehat{\mathcal{G H}}(D)$ does not depend on the position of the $X$-marking. Since we can always change them in a way that $D$ becomes a diagram for an unlink with a special $O$-marking on every component, the claim follows from the results in the previous subsection.

Even though in this case the homology is no longer a link invariant, we can still prove the following theorem.

Theorem 3.3 Let us consider two grid diagrams $D_{1}$ and $D_{2}$ representing smoothly isotopic links $L_{1}$ and $L_{2}$ such that all the isotopic components both contain or not contain a special $O$-marking. Then we have that $\widehat{\mathcal{G H}}\left(D_{1}\right)$ is filtered isomorphic to $\widehat{\mathcal{G H}}\left(D_{2}\right)$ and the isomorphism preserves the Maslov grading. Hence, we can denote the homology group of an $n$-component link $L$ with $\widehat{\mathcal{G H}}(L)$ and it depends only on which components of the link contain a special $O$-marking. 
Proof Lemma 4.1 in [14] tells us that such two grid diagrams differ by a finite sequence of grid moves: reordering of the $O$-markings, commutations and stabilizations. See Section 3 in [14] for more details. Then it is enough to prove the theorem in the case when $D_{2}$ is obtained from $D_{1}$ by one of these three moves.

Applying the results in [7] Chapter 5 and 13 we find filtered quasi-isomorphisms for each move and this implies $\widehat{\mathcal{G H}}\left(D_{1}\right) \cong \widehat{\mathcal{G H}}\left(D_{2}\right)$.

We use the homology groups $\widehat{\mathcal{G H}}^{\mathbb{Q}}(L)$ to define some cobordism maps in Section 4.

\subsection{Symmetries}

\subsubsection{Reversing the orientation}

If $-L$ is the link obtained from $L$ by reversing the orientation of all the components then

$$
T_{-L}(d, s)=T_{L}(d, s) \quad \text { for any } d, s \in \mathbb{Z}
$$

and $\tau(-L)=\tau(L)$.

To see this, consider a grid diagram $D$ of $L$, then it is easy to observe that, if we reflect $D$ along the diagonal going from the top-left to the bottom-right of the grid, the diagram $D^{\prime}$ obtained in this way represents $-L$. Hence, we take the map $\Phi: S(D) \rightarrow S\left(D^{\prime}\right)$ that sends a grid state $x$ into its reflection $x^{-}$and now, from Proposition 4.3.1 in [7], we have that $M\left(x^{-}\right)=M(x)$ and $A\left(x^{-}\right)=A(x)$. This means that $\Phi$ is a filtered quasi-isomorphism between $\widehat{G C}(D)$ and $\widehat{G C}\left(D^{\prime}\right)$, since clearly the differentials commute with $\Phi$. This gives that $\widehat{\mathcal{G H}}(-L) \cong \widehat{\mathcal{G H}}(L)$ and then Equation (7) follows.

\subsubsection{Mirror image}

For an $n$-component link $L$ we have that the function $T$ of the mirror image $L^{*}$ is given by the following equation

$$
T_{L^{*}}(d, s)=T_{L}(-d+1-n,-s) \quad \text { for any } d, s \in \mathbb{Z} .
$$

The proof of this relation is similar to the proof of Proposition 7.1.2 in [7]. First, given a complex $\mathcal{C}$ with a filtration $\mathcal{F}$, we introduce the filtered dual complex $\mathcal{C}^{*}$, equipped with a filtration $\mathcal{F}^{*}$ by taking

$$
\left(\mathcal{F}^{*}\right)^{s}\left(\mathcal{C}^{*}\right)_{d}=\operatorname{Ann}\left(\mathcal{F}^{-s-1} \mathcal{C}_{-d}\right) \subset\left(\mathcal{C}_{-d}\right)^{*}=\left(\mathcal{C}^{*}\right)_{d} \quad \text { for any } d, s \in \mathbb{Z},
$$

where $\operatorname{Ann}\left(\mathcal{F}^{h} \mathcal{C}_{k}\right)$ is the subspace of $\left(\mathcal{C}_{k}\right)^{*}$ consisting of all the linear functionals that are zero over $\mathcal{F}^{h} \mathcal{C}_{k}$.

Second, given a grid diagram $D$ of $L$, we call $(\widetilde{G C}(D), \widetilde{\partial})$ the filtered fully blocked chain complex $\frac{(\widehat{G C}(D), \widehat{\partial})}{V_{1}=\ldots=V_{m}=0}$ and we also denote with $W$ the two dimensional $\mathbb{F}$-vector space with generators in grading and minimal level $(d, s)=(0,0)$ and $(d, s)=(-1,-1)$. We want to prove the following proposition.

Proposition 3.4 $\widehat{\mathcal{G H}}\left(L^{*}\right) \cong \widehat{\mathcal{G H}}^{*}(L) \llbracket 1-n, 0 \rrbracket$, where the filtration on $\widehat{\mathcal{G H}}^{*}(L)$ is $\mathcal{F}^{*}$. 
Proof Let $D^{*}$ be the diagram obtained by reflecting $D$ through a horizontal axis. The diagram $D^{*}$ represents $L^{*}$. Reflection induces a bijection $x \rightarrow x^{*}$ between grid states for $D$ and those for $D^{*}$, inducing a bijection between empty rectangles in $\operatorname{Rect}^{\circ}(x, y)$ and empty rectangles in $\operatorname{Rect}^{\circ}\left(y^{*}, x^{*}\right)$. Hence, the reflection induces a filtered isomorphism

$$
\widetilde{G C}\left(D^{*}\right) \cong \widetilde{G C}^{*}(D) \llbracket 1-\operatorname{grd}(D), n-\operatorname{grd}(D) \rrbracket,
$$

where the shifts are given by the fact that $M\left(x^{*}\right)=-M(x)+1-\operatorname{grd}(D)$ and $A\left(x^{*}\right)=-A(x)+n-$ $\operatorname{grd}(D)$.

Now, from Lemma 14.1.11 in [7], we have the filtered quasi-isomorphism

$$
\widetilde{G C}(D) \cong \widehat{G C}(D) \otimes W^{\otimes(\operatorname{grd}(D)-n)} .
$$

Combining these two relations and observing that

$$
\left(W^{*}\right)^{\otimes(\operatorname{grd}(D)-n)} \cong W^{\otimes(\operatorname{grd}(D)-n)} \llbracket \operatorname{grd}(D)-n, \operatorname{grd}(D)-n \rrbracket
$$

leads to the filtered quasi-isomorphism

$$
\widehat{G C}\left(D^{*}\right) \cong \widehat{G C}^{*}(D) \llbracket 1-n, 0 \rrbracket .
$$

Proposition 3.4 says that $\mathcal{F}^{s} \widehat{\mathcal{G H}}_{d}\left(L^{*}\right) \cong\left(\mathcal{F}^{*}\right)^{s}\left(\widehat{\mathcal{G H}}^{*}\right)_{d-1+n}(L)$ for every $d, s \in \mathbb{Z}$. Then we can prove Equation (8):

$$
\begin{aligned}
T_{L^{*}}(d, s) & =\operatorname{dim} \frac{\left(\mathcal{F}^{*}\right)^{s}\left(\widehat{\mathcal{G H}}^{*}\right)_{d-1+n}(L)}{\left(\mathcal{F}^{*}\right)^{s-1}\left(\widehat{\mathcal{G H}}^{*}\right)_{d-1+n}(L)}=\operatorname{dim} \frac{\operatorname{Ann}\left(\mathcal{F}^{-s-1} \widehat{\mathcal{G H}}_{-d+1-n}(L)\right)}{\operatorname{Ann}\left(\mathcal{F}^{-s} \widehat{\mathcal{G H}}_{-d+1-n}(L)\right)}= \\
& =\operatorname{dim} \frac{\mathcal{F}^{-s} \widehat{\mathcal{G H}}_{-d+1-n}(L)}{\widehat{\mathcal{F}}^{-s-1} \widehat{\mathcal{G H}}_{-d+1-n}(L)}=T_{L}(-d+1-n,-s) .
\end{aligned}
$$

If we define $\tau^{*}(L)$ as the unique integer such that $T_{L}\left(1-n, \tau^{*}(L)\right)=1$ then we have proved that

$$
\tau\left(L^{*}\right)=-\tau^{*}(L) .
$$

In particular for a knot $K$, where $\tau^{*}(K)=\tau(K)$, we have $\tau\left(K^{*}\right)=-\tau(K)$. Moreover, we have the following corollary.

Corollary 3.5 Suppose that $L$ is smoothly isotopic to $L^{*}$. Then,

$$
T_{L^{*}}(d, s)=T_{L}(d, s) \quad \text { for any } d, s \in \mathbb{Z}
$$

and so Equation (8) gives that the function $T_{L}$ has a central symmetry in the point $\left(\frac{1-n}{2}, 0\right)$. In particular $\tau^{*}(L)=-\tau(L)$ and, for knots, $\tau(K)=0$.

\subsubsection{Connected sum}

Given two links $L_{1}$ and $L_{2}$, the function $T$ of the connected sum $L_{1} \# L_{2}$ is the convolution product of the $T$ functions of $L_{1}$ and $L_{2}$; in other words

$$
T_{L_{1} \# L_{2}}(d, s)=\sum_{\substack{d=d_{1}+d_{2} \\ s=s_{1}+s_{2}}} T_{L_{1}}\left(d_{1}, s_{1}\right) \cdot T_{L_{2}}\left(d_{2}, s_{2}\right) \quad \text { for any } d, s \in \mathbb{Z} .
$$


This equation is very hard to prove in the grid diagram settings, but it has been proved quite easily using the holomorphic definition of link Floer Homology. In fact Ozsváth and Szabó proved that, if $D$ is a Heegaard diagrams for $L$ and $\widehat{C F L}(D)$ denotes the link Floer complex, there is a filtered chain homotopy equivalence

$$
\widehat{C F L}\left(D_{1}\right) \otimes \widehat{C F L}\left(D_{2}\right) \longrightarrow \widehat{C F L}\left(D_{1} \# D_{2}\right)
$$

which gives that $\widehat{\mathcal{G H}}\left(L_{1} \# L_{2}\right) \cong \widehat{\mathcal{G H}}\left(L_{1}\right) \otimes \widehat{\mathcal{G H}}\left(L_{2}\right)$. See Section 7 in [8].

We see immediately that the homology and the $T$ function of $L_{1} \# L_{2}$ are independent from the choice of the components used to perform the connected sum; moreover the $\tau$-invariant is additive:

$$
\tau\left(L_{1} \# L_{2}\right)=\tau\left(L_{1}\right)+\tau\left(L_{2}\right) .
$$

\subsubsection{Disjoint union}

The disjoint union of two links $L_{1}$ and $L_{2}$ is equivalent to $L_{1} \#\left(L_{2} \sqcup \bigcirc\right)$. Thus by Equation (6) and (11) we have the following relation:

$$
T_{L_{1} \sqcup L_{2}}(d, s)=\sum_{\substack{d=d_{1}+d_{2} \\ s=s_{1}+s_{2}}} T_{L_{1}}\left(d_{1}, s_{1}\right) \cdot\left(T_{L_{2}}\left(d_{2}, s_{2}\right)+T_{L_{2}}\left(d_{2}+1, s_{2}\right)\right) \quad \text { for any } d, s \in \mathbb{Z},
$$

or in other words: $\widehat{\mathcal{G H}}\left(L_{1} \sqcup L_{2}\right) \cong \widehat{\mathcal{G H}}\left(L_{1}\right) \otimes \widehat{\mathcal{G H}}\left(L_{2}\right) \otimes V$, where $V$ is the two dimensional $\mathbb{F}$-vector space with generators in grading and minimal level $(d, s)=(-1,0)$ and $(d, s)=(0,0)$. We have immediately that

$$
\tau\left(L_{1} \sqcup L_{2}\right)=\tau\left(L_{1} \# L_{2}\right)=\tau\left(L_{1}\right)+\tau\left(L_{2}\right)
$$

\subsubsection{Quasi-alternating links}

We recall that quasi-alternating links are the smallest set of links $\mathcal{Q}$ that satisfies the two properties:

(1) The unknot is in $\mathcal{Q}$.

(2) $L$ is in $\mathcal{Q}$ if it admits a diagram with a crossing whose two resolutions $L_{0}$ and $L_{1}$ are both in $\mathcal{Q}, \operatorname{det}\left(L_{i}\right) \neq 0$ and $\operatorname{det}\left(L_{0}\right)+\operatorname{det}\left(L_{1}\right)=\operatorname{det}(L)$.

The above definition and Lemma 3.2 in [9] imply that every quasi-alternating link is non-split and every non-split alternating link is quasi-alternating. Moreover, quasi-alternating links are both Khovanov and link Floer homology thin, which means that their homologies are supported in two and one lines respectively, and the homology is completely determined by the signature and the Jones (Alexander in the hat version of link Floer homology) polynomial. The following proposition says that the same is true in filtered grid homology.

Theorem 3.6 If $L$ is an $n$-component quasi-alternating link then the function $T_{L}$ is supported in a line; more specifically the following relation holds:

$$
T_{L}(d, s) \neq 0 \quad \text { if and only if } \quad s=d+\frac{n-1-\sigma(L)}{2} \quad \text { for } 1-n \leqslant d \leqslant 0
$$

where $\sigma(L)$ is the signature of $L$. 
Proof We already know that if $T_{L}(d, s) \neq 0$ then $1-n \leqslant d \leqslant 0$, so we only have to prove the alignment part of the statement.

Take a grid diagram $D$ for $L$, then from Theorem 10.3.3 in [7] the claim is true for the bigraded homology $H_{*, *}(\operatorname{gr}(\widehat{G C}(D))) \cong \widehat{G H}(L)$. Since $T_{L}(d, s) \neq 0$ implies that $H_{d, s}(\operatorname{gr}(\widehat{G C}(D)))$ is non zero, the theorem follows.

From Theorem 3.6 we obtain immediately the following corollary.

Corollary 3.7 If $L$ is an $n$-component quasi-alternating link then $\tau(L)=\frac{n-1-\sigma(L)}{2}$ and $\tau\left(L^{*}\right)=$ $n-1-\tau(L)$.

\section{Cobordisms}

\subsection{Induced maps and degree shift}

In this section we study the behaviour of the function $T$ under cobordisms. A genus $g$ cobordism between two links $L_{1}$ and $L_{2}$ is a smooth embedding $f: \Sigma_{g} \rightarrow S^{3} \times I$ where $\Sigma_{g}$ is a compact orientable surface of genus $g$ (more precisely $\Sigma_{g}$ has connected components $\Sigma_{g_{1}}, \ldots, \Sigma_{g_{J}}$ and it is $\left.g=g_{1}+\ldots+g_{J}\right)$ such that

(1) $f\left(\partial \Sigma_{g}\right)=\left(-L_{1}\right) \times\{0\} \sqcup L_{2} \times\{1\}$.

(2) $f\left(\Sigma_{g} \backslash \partial \Sigma_{g}\right) \subset S^{3} \times(0,1)$.

(3) Every connected component of $\Sigma_{g}$ has boundary in both $L_{1}$ and $L_{2}$.

In all the figures in this section cobordisms are drawn as standard surfaces in $S^{3}$, but they can be knotted in $S^{3} \times I$.

Some of the induced maps that appear in this subsection come from the work of Sucharit Sarkar in [14]; though the grading shifts are different, because Sarkar used a different definition of the Alexander grading, ignoring the number of component of the link.

It is a standard result in Morse theory that a link cobordism can be decomposed into five standard cobordisms. We find maps in homology for each case. From now on, given a link $L_{i}$, we denote with $D_{i}$ one of its grid diagrams.

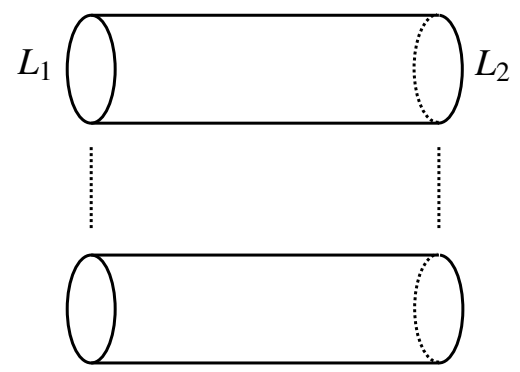

Figure 4: Identity cobordism. 
i) Identity cobordism. This cobordism, with no critical points (Figure 4), represents a sequence of Reidemeister moves; in other words $L_{1}$ and $L_{2}$ are smoothly isotopic. At the end of Section 2 we remarked that filtered homology is a link invariant; more precisely what we have is a filtered quasi-isomorphism between $\widehat{G C}\left(D_{1}\right)$ and $\widehat{G C}\left(D_{2}\right)$. This, as we know, induces a filtered isomorphism in homology.

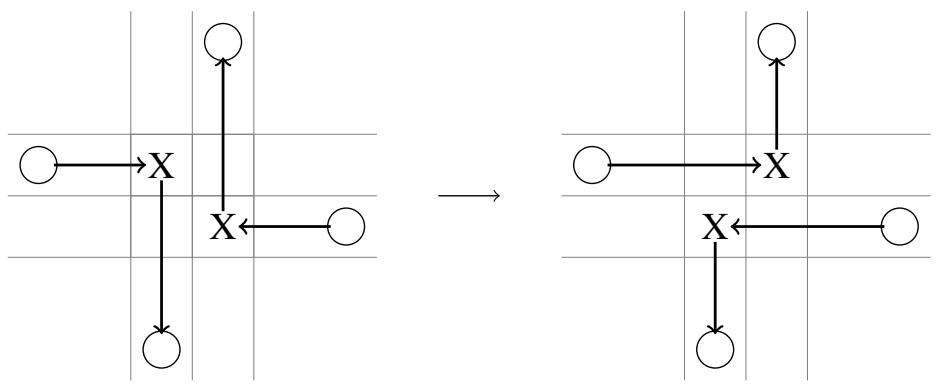

Figure 5: Band move in a grid diagram.

ii) Split cobordism. This cobordism (right in Figure 6) represents a band move when $L_{2}$ has one more component than $L_{1}$. Take $D_{1}$ with a $2 \times 2$ square with two $X$-markings, one at the top-left and one at the bottom-right; then we claim that $D_{2}$ is obtained from $D_{1}$ by deleting this two $X$-markings and putting two new ones: at the top-right and the bottom-left, as shown in Figure 5. In order to construct the complex $\widehat{G C}\left(D_{2}\right)$ we need to create one more special
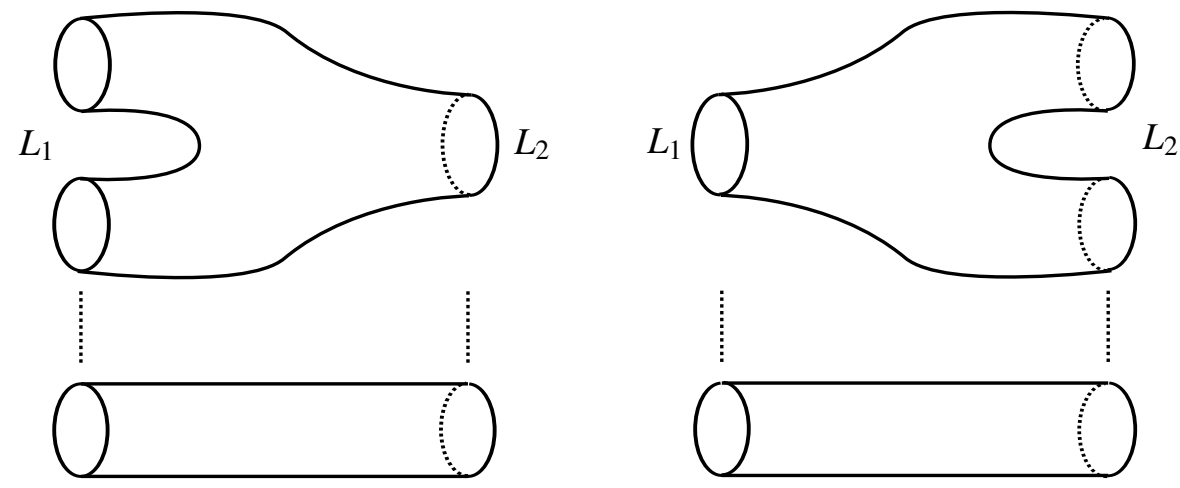

Figure 6: Merge and split cobordisms.

$O$-marking on the new component of $L_{2}$. To avoid this problem we first consider the identity map in the filtered $\widetilde{G C}$ theory

$$
\text { Id : } \widetilde{G C}\left(D_{1}\right) \longrightarrow \widetilde{G C}\left(D_{2}\right),
$$

which clearly is a chain map since now every $O$-marking is special; moreover it induces an isomorphism in homology, that preserves the Maslov grading, and a direct computation gives that it is filtered of degree 1 .

Now we use Equation (9) and we get an isomorphism

$$
\Phi_{\text {Split }}: \widehat{\mathcal{G H}}\left(L_{1}\right) \otimes W \longrightarrow \widehat{\mathcal{G H}}\left(L_{2}\right)
$$

that is a degree 1 filtered map which still preserves the Maslov grading. The $W$ factor appears because in Equation (9) we take into account the size of $D_{i}$ and the number of components of 
$L_{i}$; while the first quantity is the same for both diagrams the link $L_{2}$ has one more component than $L_{1}$.

iii) Merge cobordism. This cobordism (left in Figure 6) represents a band move when $L_{1}$ has one more component than $L_{2}$. We have an isomorphism

$$
\Phi_{\text {Merge }}: \widehat{\mathcal{G H}}\left(L_{1}\right) \longrightarrow \widehat{\mathcal{G H}}\left(L_{2}\right) \otimes W .
$$

The map is obtained in the same way as $\Phi_{\text {Split }}$ in the previous case, but with the difference that now it is filtered of degree 0 .

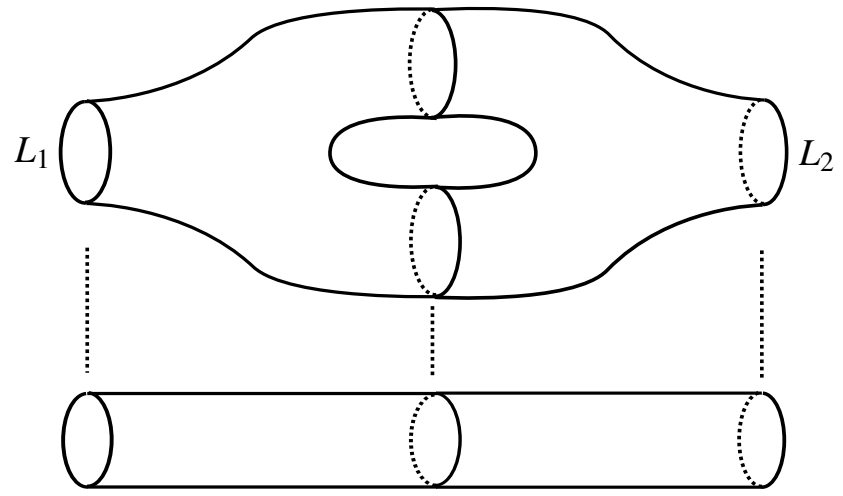

Figure 7: Torus cobordism.

Sometimes we are more interested in when a split and a merge cobordism appear together, the second just after the first, in the shape of what we call a torus cobordism (Figure 7). We have the following proposition.

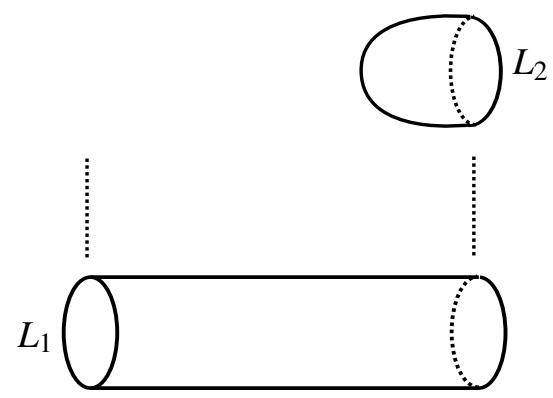

Figure 8: Birth cobordism.

Proposition 4.1 Let $\Sigma$ be a torus cobordism between two links $L_{1}$ and $L_{2}$. Then $\Sigma$ induces a Maslov grading preserving isomorphism between $\widehat{\mathcal{G H}}\left(L_{1}\right)$ and $\widehat{\mathcal{G H}}\left(L_{2}\right)$, which is filtered of degree 1 .

Proof We can choose $D_{1}$ in a way that the split and the merge band moves can be performed on two disjoint bands. Then we apply twice the move shown in Figure 5 and we take as map the identity. In this case the identity is a chain map because $L_{2}$ has the same number of components of $L_{1}$; this means that the special $O$-markings in $D_{1}$ and $D_{2}$ are the same and then the two differentials coincide. In this way we obtain an isomorphism in homology with Maslov grading shift and filtered degree equal to the sum of the ones in ii) and iii). 
iv) Birth cobordism. A cobordism (Figure 8) representing a birth move.

Since our cobordisms have boundary in both $L_{1}$ and $L_{2}$, we can always assume that a birth move is followed (possibly after some Reidemeister moves) by a merge move. Thus it is enough to define a map for the composition of these three cobordisms and this is what we do in the following proposition.

Proposition 4.2 Let $\Sigma$ be a cobordism between two links $L_{1}$ and $L_{2}$ like the one in Figure 9. Then

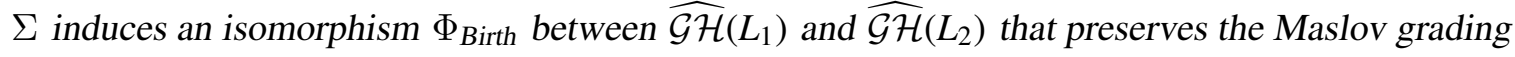
and it is filtered of degree 0 .

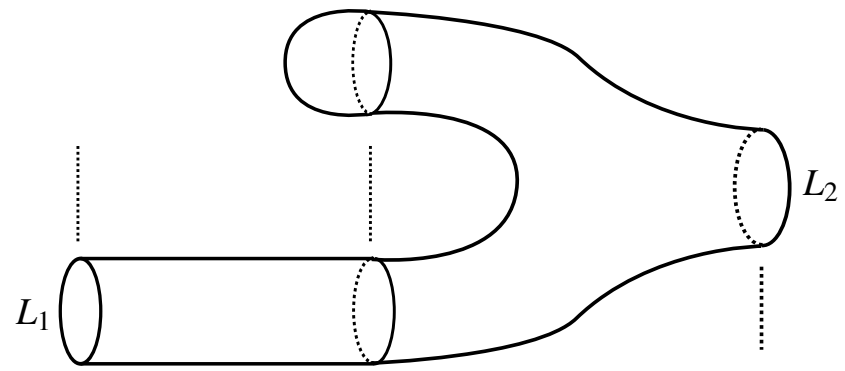

Figure 9: A more useful birth cobordism.

Proof The first step is to construct a map $s_{1}$ associated to the grid move shown in Figure 10; note that we add a normal $O$-marking, since later we merge the new unknot component with an already exisiting one. Let us denote with $D_{1}^{\prime}$ the stabilized diagram and with $c=\alpha \cap \beta$ the point in the picture; then we have the inclusion $i: S\left(D_{1}\right) \rightarrow S\left(D_{1}^{\prime}\right)$ that sends a grid state $x$ in $D_{1}$ to the grid state in $D_{1}^{\prime}$ constructed from $x$ by adding the point $c$. Then $s_{1}: \widehat{G C}\left(D_{1}\right) \rightarrow \widehat{G C}\left(D_{1}^{\prime}\right)$ is defined by the following formula:

$$
s_{1}(x)=\sum_{y \in S\left(D_{1}^{\prime}\right)} \sum_{\substack{H \in \mathcal{S} \mathcal{L}(i(x), y, c) \\ H \cap s \mathbb{O}=\emptyset}} V_{1}^{n_{1}(H)} \cdot \ldots \cdot V_{m}^{n_{m}(H)} y \quad \text { for any } x \in S\left(D_{1}\right)
$$

where $\mathcal{S} \mathcal{L}(x, z, p)$ is the set of all the snail-like domains (the exact definition can be found in [7]

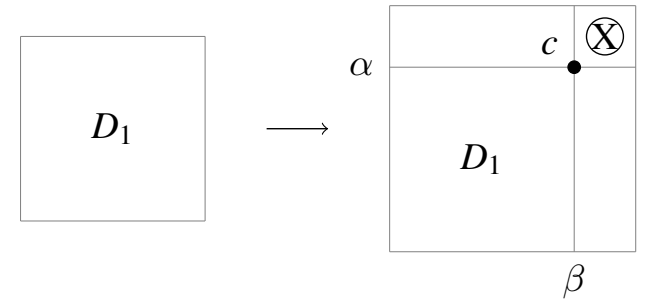

Figure 10: Birth move in a grid diagram.

Chapter 13) centered at $p$ joining $x$ to $z$, illustrated in Figure $11 ; n_{i}(H)$ is the number of times $H$ passes through $O_{i}$ and $m$ is the number of the normal $O$-markings of $D_{1}$. In [5] is proved that $s_{1}$ is a filtered quasi-isomorphism which induces a filtered isomorphism between $\widehat{\mathcal{G H}}\left(L_{1}\right) \rightarrow$ $\widehat{\mathcal{G H}}^{\mathbb{O}}\left(L_{1} \sqcup \bigcirc\right)$; where the special $O$-markings on $L_{1} \sqcup \bigcirc$ coincide with the ones on $L_{1}$ (the new 
unknotted component has a normal $O$-marking). Moreover, in [14] Sarkar showed that the map $s_{1}$ is filtered of degree 0 .

At this point we compose $s_{1}$ with the map $s_{2}$ given by the Reidemeister moves, which is a filtered quasi-isomorphism for Theorem 3.3, and finally with $s_{3}$, the identity associated to the band move of Figure 5. The map $s_{3}$ induces an isomorphism $\widehat{\mathcal{G H}}\left(L_{1} \sqcup \bigcirc\right) \rightarrow \widehat{\mathcal{G H}}\left(L_{2}\right)$ that clearly preserves
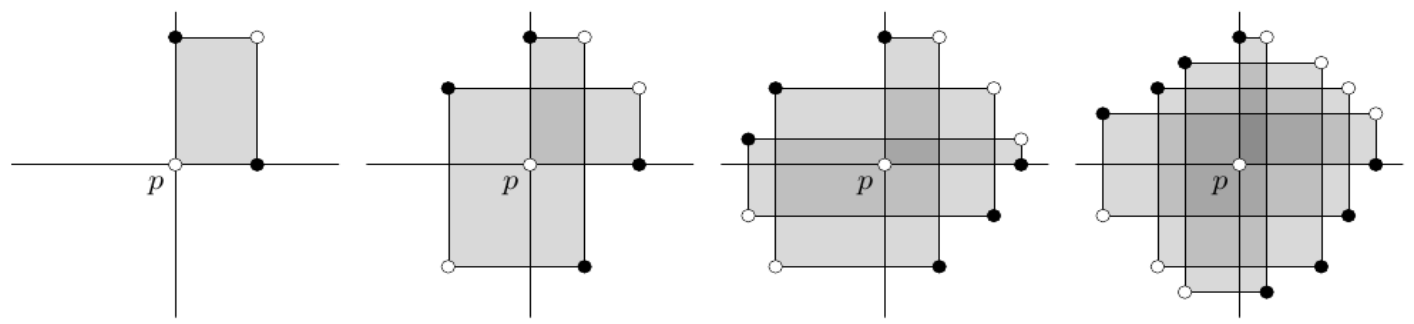

Figure 11: Some of the snail-like domains $\mathcal{S L}(x, z, p)$ : the coordinates of $x$ and $z$ are represented by the white and black circles.

the Maslov grading and again we easy compute that it is filtered of degree 0 .

Hence, the composition of these three maps that we defined induces the isomorphism in the claim.

v) Death cobordism. This cobordism (Figure 12) represents a death move. Since this move can also be seen as a birth move between $L_{2}^{*}$ and $L_{1}^{*}$, we take the dual map of

$$
\Phi_{\text {Birth }}: \widehat{\mathcal{G H}}\left(L_{2}^{*}\right) \longrightarrow \widehat{\mathcal{G H}}\left(L_{1}^{*}\right)
$$

which exists from Proposition 4.2; then $\Phi_{\text {Birth }}^{*}=\Phi_{\text {Death }}$, by Proposition 3.4, is a map between $\widehat{\mathcal{G H}}\left(L_{1}\right)$ and $\widehat{\mathcal{G H}}\left(L_{2}\right)$. Furthermore, it is still an isomorphism that is filtered of degree 0 and preserves the Maslov grading.

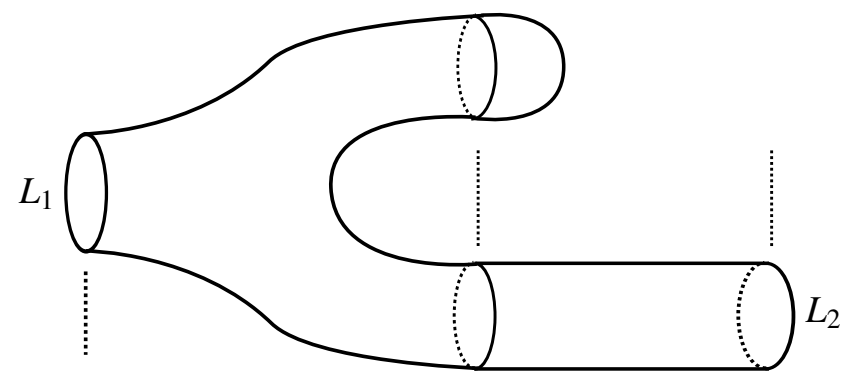

Figure 12: Death cobordism.

The results for birth and death cobordisms in this section immediately give the following corollary.

Corollary 4.3 Suppose there is a birth or a death cobordism as in Figures 9 and 12 between two links $L_{1}$ and $L_{2}$. Then we have that $\widehat{\mathcal{G H}}\left(L_{1}\right) \cong \widehat{\mathcal{G H}}\left(L_{2}\right)$. 


\subsection{Strong concordance invariance}

We want to prove Theorem 1.2. We remark that a strong cobordism is a cobordism $\Sigma$, between two links with the same number of components, such that every connected component of $\Sigma$ is a knot cobordism between a component of the first link and one of the second link. Moreover, if the connected components of $\Sigma$ are all annuli then we call $\Sigma$ a strong concordance. We start by observing that Proposition 4.1 leads to the following corollary.

Corollary 4.4 Suppose there is a strong cobordism $\Sigma$ between $L_{1}$ and $L_{2}$ such that $\Sigma$ is the composition of $g(\Sigma)$ torus cobordisms, not necessarily all of them belonging to the same component of $\Sigma$. Then $\Sigma$ induces an isomorphism between $\widehat{\mathcal{G H}}\left(L_{1}\right)$ and $\widehat{\mathcal{G H}}\left(L_{2}\right)$, which is filtered of degree $g(\Sigma)$ and preserves the Maslov grading.

Now, if we have an isomorphism $F: \widehat{\mathcal{G H}}\left(L_{1}\right) \rightarrow \widehat{\mathcal{G H}}\left(L_{2}\right)$ that preserves the Maslov grading and it is filtered of degree $t$, which means that there are inclusions $F\left(\mathcal{F}^{s} \widehat{\mathcal{G H}}_{d}\left(L_{1}\right)\right) \subset \mathcal{F}^{s+t} \widehat{\mathcal{G H}}_{d}\left(L_{2}\right)$ for every $d, s \in \mathbb{Z}$, then $\tau\left(L_{2}\right) \leqslant \tau\left(L_{1}\right)+t$. Hence we can prove the following theorem that immediately implies the invariance statement.

Theorem 4.5 Suppose that $\Sigma$ is a strong cobordism between two links $L_{1}$ and $L_{2}$. Then

$$
\left|\tau\left(L_{1}\right)-\tau\left(L_{2}\right)\right| \leqslant g(\Sigma) .
$$

Furthermore, if $L_{1}$ and $L_{2}$ are strongly concordant then $\widehat{\mathcal{G H}}\left(L_{1}\right) \cong \widehat{\mathcal{G H}}\left(L_{2}\right)$.

Proof By Proposition B.5.1 in [7] we can suppose that, in $\Sigma$, 0-handles come before 1-handles while 2-handles come later; moreover we can say that $\Sigma$ is the composition of birth, torus and death cobordisms (and obviously some identity cobordisms). Each of these induces an isomorphism in homology that also respects the Maslov grading.

For the first part, we only need to check what is the filtered degree of the isomorphism between $\widehat{\mathcal{G H}}\left(L_{1}\right)$ and $\widehat{\mathcal{G H}}\left(L_{2}\right)$, obtained by the composition of all the induced maps on each piece of $\Sigma$. Birth, death and identity are filtered of degree 0 , while, from Corollary 4.4 , the torus cobordisms are filtered of degree $g(\Sigma)$. Then we obtain

$$
\tau\left(L_{2}\right) \leqslant \tau\left(L_{1}\right)+g(\Sigma) .
$$

For the other inequality we consider the same cobordism, but this time from $L_{2}$ to $L_{1}$.

Now, for the second part, we observe that now there are no torus cobordisms and then the claim follows from Corollary 4.3.

\subsection{A lower bound for the slice genus}

Suppose $\Sigma$ is a cobordism (not necessarily strong) between two links $L_{1}$ and $L_{2}$. Denote with $\Sigma_{1}, \ldots, \Sigma_{J}$ the connected components of $\Sigma$. For $i=1,2$ we define the integers $l_{i}^{k}(\Sigma)$ as the number of components of $L_{i}$ that belong to $\Sigma_{k}$ minus 1 ; in particular $l_{i}^{k}(\Sigma) \geqslant 0$ for any $k, i$. Finally, we say that $l_{i}(\Sigma)=\sum_{k=1}^{J} l_{i}^{k}(\Sigma)=n_{i}-J$, where $n_{i}$ is the number of component of $L_{i}$. For example, if $\Sigma$ is the cobordism in Figure 13 then $J=2$, ordering $\Sigma_{1}$ and $\Sigma_{2}$ from above to bottom, we have that $l_{1}(\Sigma)=3$ and $l_{2}(\Sigma)=4$; while $l_{1}^{1}(\Sigma)=2, l_{1}^{2}(\Sigma)=1, l_{2}^{1}(\Sigma)=3$ and $l_{2}^{2}(\Sigma)=1$. We have the following lemma. 
Lemma 4.6 If $\Sigma$ has no 0,2-handles then, up to rearranging 1-handles, we can suppose that $\Sigma$ is like in Figure 13: there are $l_{1}(\Sigma)$ merge cobordisms between $\left(0, t_{1}\right), l_{2}(\Sigma)$ split cobordisms between $\left(t_{2}, 1\right)$ and $g(\Sigma)$ torus cobordisms between $\left(t_{1}, t_{2}\right)$. We have no other 1-handles except for the ones we considered before.

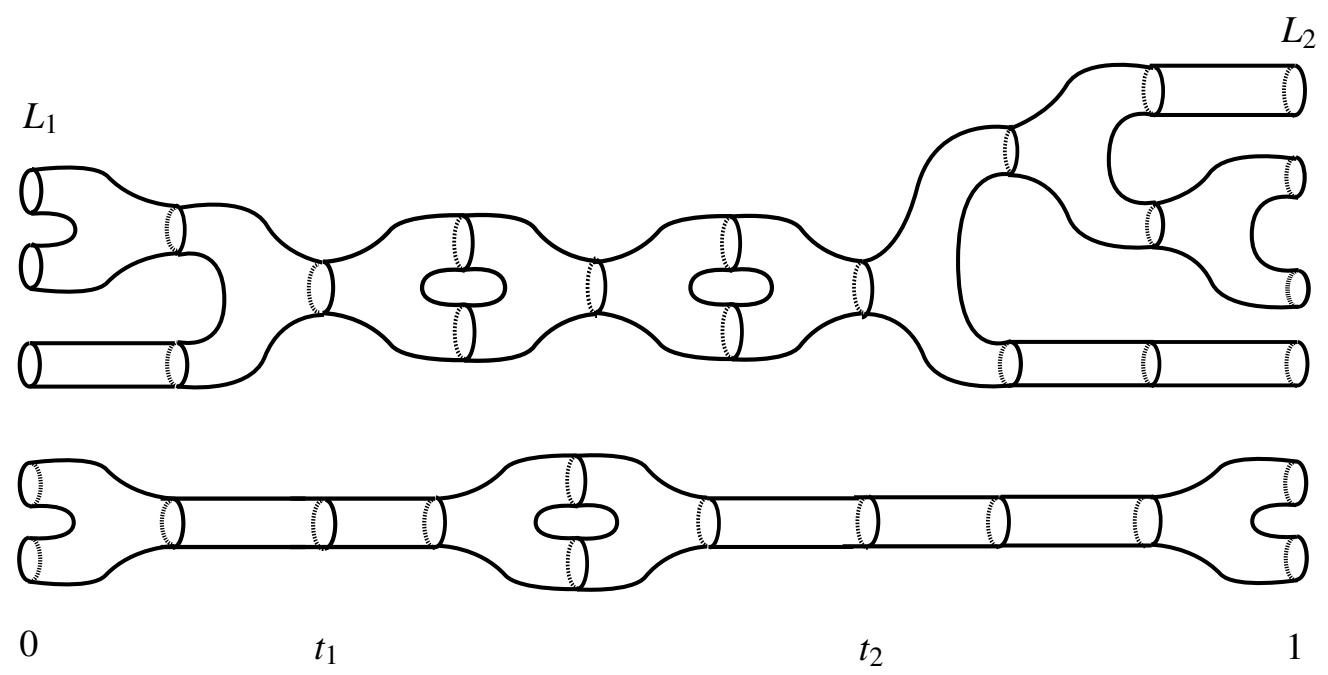

Figure 13

Proof We consider a connected component $\Sigma_{k}$, which is a cobordism between $L_{1}^{k}$ and $L_{2}^{k}$, and we fix a Morse function $f: S^{3} \times[0,1] \rightarrow[0,1]$. After some Reidemeister moves, by Proposition B.5.1 we can assume that all the band moves are performed on disjoint bands, in particular we can apply them in every possible order.

Since $\Sigma_{k}$ has boundary in both $L_{1}^{k}$ and $L_{2}^{k}$ by the definition of cobordism given at beginning of Section 4 , if we take an ordering for the components of $L_{1}^{k}$, we can find a merge cobordism joining the first and the second component of $L_{1}^{k}$ at some point $\bar{t}$ in $(0,1)$; we assume that the associated band move is the first we apply on $L_{1}^{k}$. Now we just do the same thing on the other components, but taking the new component instead of the first two. In this way we have that there is a $t_{1} \in(0,1)$ such that $\Sigma_{k} \cap f^{-1}\left[0, t_{1}\right]$ is composed by $l_{1}^{k}(\Sigma)$ merge cobordisms and $\Sigma_{k} \cap f^{-1}\left(t_{1}\right)$ is a knot.

In the same way we find that, for a certain $t_{2} \in(0,1)$, the cobordism $\Sigma_{k} \cap f^{-1}\left[t_{2}, 1\right]$ is composed by $l_{2}^{k}(\Sigma)$ split cobordisms and $\Sigma_{k} \cap f^{-1}\left(t_{2}\right)$ is a knot.

At this point $\Sigma_{k} \cap f^{-1}\left[t_{1}, t_{2}\right]$ is a knot cobordism of genus $g\left(\Sigma_{k}\right)$ and from Lemma B.5.3 in [7] (see also [4]) we can rearrange the saddles to obtain a composition of $g\left(\Sigma_{k}\right)$ torus cobordisms like in Figure 13.

To see that there are no other 1-handles left it is enough to compute the Euler characteristic of $\Sigma_{k}$ :

$$
2-2 g\left(\Sigma_{k}\right)-l_{1}^{k}(\Sigma)-1-l_{2}^{k}(\Sigma)-1=\chi\left(\Sigma_{k}\right)=-\# \mid 1 \text {-handles } \mid .
$$

This means that the number of 1-handles in $\Sigma_{k}$ is precisely $2 g\left(\Sigma_{k}\right)+l_{1}^{k}(\Sigma)+l_{2}^{k}(\Sigma)$.

From Figure 13 we realize that merge and split cobordisms can appear alone in $\Sigma$ and not always in pair like in strong cobordisms. In case ii) and iii) of Subsection 4.1 we see that they do not induce 
isomorphisms in homology, but we find maps $\Phi_{\text {Split }}$ and $\Phi_{\text {Merge }}$ that are indeed isomorphisms if restricted to $\widehat{\mathcal{G H}}_{0}\left(L_{1}\right) \rightarrow \widehat{\mathcal{G H}}_{0}\left(L_{2}\right)$; moreover, the filtered degree is 1 for split cobordisms and 0 for merge cobordisms. Since we are looking for informations on $\tau$, this is enough for our goal and then we can prove the following inequality.

Proposition 4.7 Suppose $\Sigma$ is a cobordism between two links $L_{1}$ and $L_{2}$. Then

$$
\left|\tau\left(L_{1}\right)-\tau\left(L_{2}\right)\right| \leqslant g(\Sigma)+\max \left\{l_{1}(\Sigma), l_{2}(\Sigma)\right\} .
$$

Proof By Theorem 4.5, we can suppose that there are no 0 and no 2-handles in $\Sigma$. We can also assume that $\Sigma$ is like in Lemma 4.6.

All of these cobordisms induce isomorphisms of the homology in Maslov grading 0. The number of torus cobordisms is $g(\Sigma)$ while the number of split cobordisms (that are not part of torus cobordisms) is $l_{2}(\Sigma)$. This means that

$$
\tau\left(L_{2}\right) \leqslant \tau\left(L_{1}\right)+g(\Sigma)+l_{2}(\Sigma) .
$$

Now we do the same, but considering the cobordism going from $L_{2}$ to $L_{1}$, as we did in the proof of Theorem 4.5. We obtain that

$$
\tau\left(L_{1}\right) \leqslant \tau\left(L_{2}\right)+g(\Sigma)+l_{1}(\Sigma) .
$$

Putting the two inequalities together proves the relation in the statement of the theorem.

If $L$ is an $n$-component link, from Proposition 4.7 we have immediately Equation (1):

$$
|\tau(L)|+1-n \leqslant g_{4}(L)
$$

which, as we already said, is a lower bound for the slice genus of our link. Indeed, we can say more by using Equation (10) and observing that $g_{4}\left(L^{*}\right)=g_{4}(L)$ :

$$
\max \left\{|\tau(L)|,\left|\tau^{*}(L)\right|\right\}+1-n \leqslant g_{4}(L) .
$$

\section{The $\mathcal{G H}^{-}$version of filtered grid homology}

\subsection{A different point of view}

The collapsed filtered complex $c G C^{-}(D)$ for a grid diagram $D$ is the free $\mathbb{F}\left[V_{1}, \ldots, V_{\operatorname{grd}(D)-n}, V\right]-$ module generated by the set of grid states $S(D)$. This ring has one more variable $V$, compared to

the ring we considered for $\widehat{G C}(D)$, associated to the special $O$-markings. The differential $\partial^{-}$is defined as following:

$$
\partial^{-} x=\sum_{y \in S(D)} \sum_{r \in \operatorname{Rect}^{\circ}(x, y)} V_{1}^{O_{1}(r)} \cdot \ldots \cdot V_{m}^{O_{m}(r)} \cdot V^{O(r)} y \text { for any } x \in S(D)
$$

where $m=\operatorname{grd}(D)-n$ and $O(r)$ is the number of special $O$-markings in $r$.

It is clear from the definition that

$$
(\widehat{G C}(D), \widehat{\partial})=\frac{\left(c G C^{-}(D), \partial^{-}\right)}{V=0}
$$


The collapsed filtered unblocked homology $c \mathcal{G H}^{-}(L)$ is the homology of our new complex and it is a link invariant; but now each level $\mathcal{F}^{s} c \mathcal{G H}^{-}(L)$ has also a structure of an $\mathbb{F}[U]$-module given by $U[p]=\left[V_{i} p\right]=[V p]$ for every $i=1, \ldots, m$ and $[p] \in c \mathcal{G H}^{-}(L)$. The groups $\mathcal{F}^{s} c \mathcal{G H}_{d}^{-}(L)$ are still finite dimensional over $\mathbb{F}$ and so we can define the function $N$ as

$$
N_{L}(d, s)=\operatorname{dim}_{\mathbb{F}} \frac{\mathcal{F}^{s} c \mathcal{G} \mathcal{H}_{d}^{-}(L)}{\mathcal{F}^{s-1} c \mathcal{G} \mathcal{H}_{d}^{-}(L)} .
$$

We expect the function $N$ to be a strong concordance invariant, possibly better than $T$.

We can compute the function $N$ of the unknot:

$$
N_{\bigcirc}(d, s)=\left\{\begin{array}{cc}
1 & \text { if }(d, s)=(2 t, t), \quad t \leqslant 0 \\
0 & \text { otherwise }
\end{array}\right.
$$

and we know that

$$
c \mathcal{G} \mathcal{H}^{-}(L) \cong_{\mathbb{F}[U]} c \mathcal{G} \mathcal{H}^{-}\left(\bigcirc_{n}\right) \cong_{\mathbb{F}[U]} \mathbb{F}[U]^{2^{n-1}}
$$

as an $\mathbb{F}[U]$-module, where $n$ is the number of component of $L$.

Since $\operatorname{dim}_{\mathbb{F}} c \mathcal{G H}_{0}^{-}(L)$ is still equal to 1, we can define an invariant $\nu$ exactly like we did in Subsection 3.1 for $\tau$. A version of the $\nu$-invariant has been introduced first by Jacob Rasmussen in [12] and he proved that it is a concordance invariant for knots. In [3] Hom and $\mathrm{Wu}$ found knots whose $\nu$-invariant gives better lower bound for the slice genus than $\tau$.

Since $H_{*, *}\left(\operatorname{gr}\left(c G C^{-}(D)\right)\right)$ is isomorphic to the homology $c G H^{-}(L)$ of [7] and $N_{L}(0, \nu(L))=1$ for every diagram $D$ of $L$, we have that $c G H_{0, \nu(L)}^{-}(L)$ is non-trivial. Hence, if the homology group $c G H_{0}^{-}(L)$ is non-zero only for one Alexander grading $s$, we can argue that $\nu(L)=s$. This method can be used to compute the $\nu$-invariant of some links.

We can also define the (uncollapsed) filtered unblocked homology as the homology of the complex $\left(G C^{-}(D), \partial^{-}\right)$, where $G C^{-}(D)$ is the free $\mathbb{F}\left[V_{1}, \ldots, V_{\operatorname{grd}(D)}\right]$-module over the grid states of $D$; there are no special $O$-markings this time.

We see immediately that for every $n$-component link $L$ is

$$
\mathcal{G H}^{-}(L) \cong \mathbb{F}\left[U_{1}, \ldots, U_{n}\right]
$$

with generator in Maslov grading 0, but the filtration will of course depend on $L$.

\subsection{Proof of Theorem 1.3}

We use the complex $c G C^{-}$to prove that, for every $n$-component link $L$, an integer $s$ gives $T_{d, s}(L) \neq 0$ for some $d$ if and only if $s$ belongs to the $\tau$-set of $L$. To do this, given $\mathcal{C}$ a freely and finitely generated $\mathbb{F}[U]$-complex, we define two set of integers: $\tau(\mathcal{C})$ and $t(\mathcal{C})$. First we call $\mathcal{B}_{\tau}(\mathcal{C})$ a homogeneous, free generating set of the torsion-free quotient of $H_{*, *}(\operatorname{gr}(\mathcal{C}))$ as an $\mathbb{F}[U]$-module; then $\tau(\mathcal{C})$ is the set of $s \in \mathbb{Z}$ such that there is a $[p] \in \mathcal{B}_{\tau}(\mathcal{C})$ with bigrading $(d,-s)$ for some $d \in \mathbb{Z}$. Similarly, $t(\mathcal{C})$ is the set of the integers $s$ such that the inclusion

$$
i_{s}: \mathcal{F}^{s-1} H_{*}\left(\frac{\mathcal{C}}{U=0}\right) \longleftrightarrow \mathcal{F}^{s} H_{*}\left(\frac{\mathcal{C}}{U=0}\right)
$$

is not surjective. Note that the set $\mathcal{B}_{\tau}(\mathcal{C})$ is not unique, but $\tau(\mathcal{C})$ and $t(\mathcal{C})$ are well-defined. 
We say that $s \in \tau(\mathcal{C})$ has multiplicity $k$ if there are $k$ distinct elements in $\mathcal{B}_{\tau}(\mathcal{C})$ with bigrading $(*,-s)$, while a number $u \in t(\mathcal{C})$ has multiplicity $k$ if Coker $i_{u}$ has dimension $k$ as an $\mathbb{F}$-vector space.

Clearly, if $D$ is a grid diagram of $L, t\left(c G C^{-}(D)\right)$ is the set of the values of $s$ where the function $T_{L}$ is supported; moreover, we already remarked that $H_{*, *}\left(\operatorname{gr}\left(c G C^{-}(D)\right)\right)$ is isomorphic to $c G H^{-}(L)$ and then $\tau\left(c G C^{-}(D)\right)$ is the $\tau$-set of $L$. Hence our goal is to prove that $t\left(c G C^{-}(D)\right)=\tau\left(c G C^{-}(D)\right)$, generalizing Proposition 14.1.2 in [7].

Consider the complex

$$
\mathcal{C}=\frac{c G C^{-}(D)}{V_{1}=\ldots=V_{m}=U},
$$

where $m=\operatorname{grd}(D)-n$ and $U$ is the variable associated to the special $O$-markings. Then we define $\overline{\mathcal{C}}$ as the complex $\mathcal{C} \llbracket 1-n-m,-m \rrbracket$.

We introduce a new complex $\mathcal{C}^{\prime}=\mathcal{C} \otimes_{\mathbb{F}[U]} \mathbb{F}\left[U, U^{-1}\right]$ and we define a $\mathbb{Z} \oplus \mathbb{Z}$-filtration on $\mathcal{C}^{\prime}$ in the following way: $\mathcal{F}^{x, *} \mathcal{C}^{\prime}=U^{-x} \mathcal{C}$ for every $x \in \mathbb{Z}, \mathcal{F}^{*, y} \mathcal{C}^{\prime}=\mathcal{F}^{y} \mathcal{C}^{\prime}=\left\{p \in \mathcal{C}^{\prime} \mid A(p) \leqslant y\right\}$ for every $y \in \mathbb{Z}$ and $\mathcal{F}^{x, y} \mathcal{C}^{\prime}=\mathcal{F}^{x, *} \mathcal{C}^{\prime} \cap \mathcal{F}^{*, y} \mathcal{C}^{\prime}=U^{-x} \mathcal{F}^{y-x} \mathcal{C}$ for every $x, y \in \mathbb{Z}$. The first step is to prove that $\tau(\mathcal{C})=t(\overline{\mathcal{C}})$.

We have that

$$
\frac{\mathcal{C}}{U=0} \cong \widetilde{G C}(D) \cong \frac{\mathcal{F}^{0, *} \mathcal{C}^{\prime}}{\mathcal{F}^{-1, * \mathcal{C}^{\prime}}}
$$

moreover, for every integer $s$ we claim that

$$
\mathcal{F}^{s} \widetilde{G C}(D) \cong \frac{\mathcal{F}^{0, s} \mathcal{C}^{\prime}}{\mathcal{F}^{-1, s} \mathcal{C}^{\prime}} .
$$

Since, from Lemma 14.1.9 in [7], it is $\mathcal{F}^{x, y} \mathcal{C}^{\prime} \cong \mathcal{F}^{y, x} \overline{\mathcal{C}^{\prime}}$ for every $x, y \in \mathbb{Z}$, then we have that

$$
\mathcal{F}^{s} \frac{\overline{\mathcal{C}}}{U=0} \cong \mathcal{F}^{s} \widetilde{G C}(D) \llbracket 1-n-m,-m \rrbracket \cong \frac{\mathcal{F}^{s, 0} \mathcal{C}^{\prime}}{\mathcal{F}^{s,-1} \mathcal{C}^{\prime}} .
$$

Using this identification we obtain that $t(\overline{\mathcal{C}})$ coincides with set of $s \in \mathbb{Z}$ such that the map

$$
H_{*}\left(\frac{\mathcal{F}^{s-1,0} \mathcal{C}^{\prime}}{\mathcal{F}^{s-1,-1} \mathcal{C}^{\prime}}\right) \longleftrightarrow H_{*}\left(\frac{\mathcal{F}^{s, 0} \mathcal{C}^{\prime}}{\mathcal{F}^{s,-1} \mathcal{C}^{\prime}}\right)
$$

is not surjective.

Now we consider the complex $\operatorname{gr}(\mathcal{C})$, which is equal to $\bigoplus_{t \in \mathbb{Z}} \frac{\mathcal{F}^{0, t} \mathcal{C}^{\prime}}{\mathcal{F}^{0, t-1} \mathcal{C}^{\prime}}$. We have that the map

$$
U^{t}: \frac{\mathcal{F}^{0, t} \mathcal{C}^{\prime}}{\mathcal{F}^{0, t-1} \mathcal{C}^{\prime}} \longrightarrow \frac{\mathcal{F}^{-t, 0} \mathcal{C}^{\prime}}{\mathcal{F}^{-t,-1} \mathcal{C}^{\prime}}
$$

is an isomorphism and $\frac{\mathcal{F}^{-t, 0} \mathcal{C}^{\prime}}{\mathcal{F}^{-t,-1} \mathcal{C}^{\prime}}$ is a subspace of $\frac{\mathcal{F}^{*, 0} \mathcal{C}^{\prime}}{\mathcal{F}^{*,-1} \mathcal{C}^{\prime}}$ for every integer $t$. From Lemma 14.1.12 in [7] the latter filtered complex is isomorphic to $\frac{\operatorname{gr}(\mathcal{C})}{U=1}$, but it is also isomorphic to $\widetilde{G C}(D) \llbracket 1-n-m,-m \rrbracket$ for Equation (13). In this way we can define a surjective map

$$
\Psi: \operatorname{gr}(\mathcal{C}) \longrightarrow \frac{\operatorname{gr}(\mathcal{C})}{U=1}
$$


and it is easy to see that $\Psi\left(\mathcal{B}_{\tau}(\mathcal{C})\right)$ is still a homogeneous, free generating set of the homology; furthermore, if $[p]$ is a torsion element in $H_{*, *}(\operatorname{gr}(\mathcal{C}))$ then $[\Psi(p)]=[0]$. This means that $\tau(\mathcal{C})$ is the set of $-t \in \mathbb{Z}$ such that the map

$$
H_{*}\left(\frac{\mathcal{F}^{-t-1,0} \mathcal{C}^{\prime}}{\mathcal{F}^{-t-1,-1} \mathcal{C}^{\prime}}\right) \longleftrightarrow H_{*}\left(\frac{\mathcal{F}^{-t, 0} \mathcal{C}^{\prime}}{\mathcal{F}^{-t,-1} \mathcal{C}^{\prime}}\right)
$$

is not surjective.

If we change $-t$ with $s$ in Equation (15) then we immediately see that it coincides with Equation (14) and so $\tau(\mathcal{C})=t(\overline{\mathcal{C}})$. Moreover, we can say that an integer in $\tau(\mathcal{C})$ has multiplicity $k$ if and only if it has multiplicity $k$ in $t(\overline{\mathcal{C}})$. Finally, since the map $U^{t}$ drops the Maslov grading by $2 t$, we have that if there is a $[p] \in \mathcal{B}_{\tau}(\mathcal{C})$ with bigrading $(d, s)$ then there is a generator of $\overline{\mathcal{G H}}(D) \llbracket 1-n-m,-m \rrbracket$ with grading and minimal level $(d-2 s,-s)$.

The second and final step is to show that the previous claim implies $t\left(c G C^{-}(D)\right)=\tau\left(c G C^{-}(D)\right)$. From Lemma 14.1.11 in [7] we have the filtered quasi-isomorphisms $\mathcal{C} \cong c G C^{-}(D) \otimes W^{\otimes m}$ and $\overline{\mathcal{C}} \cong c G C^{-}(D) \otimes\left(W^{*}\right)^{\otimes m}$, where $W$ is the two dimensional $\mathbb{F}$-vector space with generators in grading and minimal level $(d, s)=(0,0)$ and $(d, s)=(-1,-1)$. Thus $t\left(c G C^{-}(D)\right)$ and $\tau\left(c G C^{-}(D)\right)$ completely determine $\tau(\mathcal{C})$ and $t(\overline{\mathcal{C}})$, so this means that they coincide and the proof is complete. Obviously, the conclusions about multiplicities and Maslov shifts are still true.

From [7] Chapter 8 we know that there is only one element $[p] \in \mathcal{B}_{\tau}\left(c G C^{-}(D)\right)$ with bigrading $\left(-2 \tau_{1},-\tau_{1}\right)$ and only another one $[q]$ with bigrading $\left(-2 \tau_{2}+1-n,-\tau_{2}\right)$. Then the proof of Theorem 1.3 implies that there are two non-zero elements in $\widehat{\mathcal{G H}}(L)$ in grading and minimal level $\left(0, \tau_{1}\right)$ and $\left(1-n, \tau_{2}\right)$. Since, from the definition of $\tau$ and $\tau^{*}$, we also know that there are only two generators of $\widehat{\mathcal{G H}}(L)$ in Maslov grading 0 and $1-n$; we have the following corollary.

Corollary 5.1 Take a grid diagram $D$ of an $n$-component link $L$ and consider a set $\mathcal{B}_{\tau}\left(c G C^{-}(D)\right)$. If $[p],[q] \in \mathcal{B}_{\tau}\left(c G C^{-}(D)\right)$ are such that $[p]$ is in bigrading $\left(-2 \tau_{1},-\tau_{1}\right)$ and $[q]$ is in bigrading $\left(-2 \tau_{2}+1-n,-\tau_{2}\right)$ then $\tau(L)=\tau_{1}$ and $\tau^{*}(L)=\tau_{2}$.

\section{Applications}

\subsection{Computation for some specific links}

In general it is hard to say when a sum of grid states is a generator of the homology, but the following lemma provides an example where we have useful information.

Lemma 6.1 Suppose $L$ is an $n$-component link with grid diagram $D$ and $x \in S(D)$ as in Figure 14. Then $[x]$ is always the generator of $\widehat{\mathcal{G H}}_{0}(L)$ and $c \mathcal{G} \mathcal{H}_{0}^{-}(L)$. Furthermore, $\tau(L)=\nu(L)=A(x)$.

Proof We show that $M(x)=0, \widehat{\partial} x=\partial^{-} x=0$ and that for every other grid state $y$ of $D$ it is $M(y) \leqslant 0$.

i) The fact that $M(x)=0$ is trivial.

ii) For every $y \in S(D)$ there are always 2 rectangles in $\operatorname{Rect}^{\circ}(x, y)$ and they contain no $O$, so they cancel when we compute the differential. 


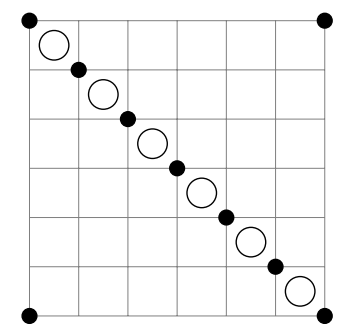

Figure 14: We denote with $x$ the grid state in the picture.

iii) We prove by induction on $\operatorname{grd}(D)$ that $M(y) \leqslant 0$ for every $y \in S(D)$.

If $\operatorname{grd}(D)=1$ then $x$ is the only grid state.

If $\operatorname{grd}(D)=2$ then there are only $x$ and $y$ and it is $M(y)=-1$.

Suppose the claim is true for the diagrams with dimension equal or smaller than $\alpha$ and let $\operatorname{grd}(D)=\alpha+1$. We denote with $I(D) \subset S(D)$ the subset of grid states that contain the point

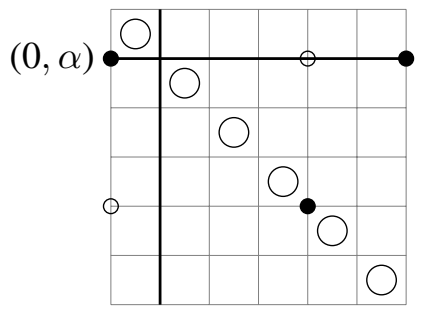

Figure 15: The state $y \in I(D)$ is marked with the black circles.

$(0, \alpha)$ as in Figure 15. Every $y \in I(D)$ is the extension of a grid state $y^{\prime}$ of the diagram $D^{\prime}$ obtained by removing the first column and the last row from $D$. By the inductive hypothesis we have $M(y)=-1+M\left(y^{\prime}\right) \leqslant-1$.

Now it easy to see that every other $z \in S(D)$ is obtained by a rectangle move from a $y \in I(D)$. Then, if $r$ is the rectangle, we have

$$
M(z)-M(y)=1-2 \cdot \#|r \cap \mathbb{O}|+2 \cdot \#|\operatorname{Int}(r) \cap y|,
$$

but \#|Int $(r) \cap y\left|\leqslant \min \left\{\pi_{1}(r), \pi_{2}(r)\right\}=\#\right| r \cap \mathbb{O} \mid$ where $\pi_{i}(r)$ is the lenght of the edges of $r$. Hence $M(z) \leqslant 0$.

In Lemma 6.1 we used that the grid diagram $D$ has all the $O$-markings aligned on a diagonal. It is easy to see that if a link admits such a diagram then it is positive. On the other hand, it seems difficult for the converse to be true.

\subsection{Torus links}

We compute the $\tau$-invariant of every torus link. Consider the grid diagram $D_{q, p}$ in Figure 16, representing the torus link $T_{q, p}$ with $q \leqslant p$ and all the components oriented in the same direction. By Lemma 6.1 we know that $[x]$ is the only generator of $\widehat{\mathcal{G H}}_{0}\left(T_{q, p}\right)$. If we denote with $n$ the number 


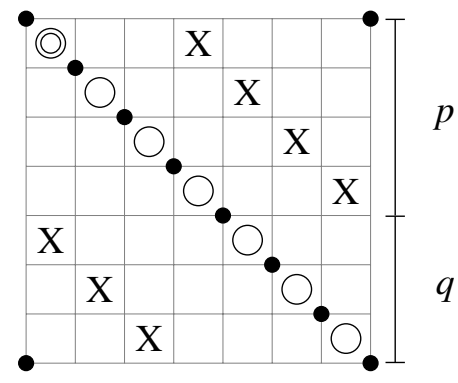

Figure 16: $x$ is the grid state in the picture.

of components of $T_{q, p}$ then a simple computation gives

$$
\begin{aligned}
A(x) & =\frac{1}{2}\left(M(x)-M_{\mathbb{X}}(x)-\operatorname{grd}\left(D_{q, p}\right)+n\right)=\frac{1}{2}\left(-M_{\mathbb{X}}(x)-p-q+n\right)= \\
& =\frac{1}{2}\left[2 \sum_{i=1}^{q-1} i+q(p-q+1)-p-q+n\right]=\frac{1}{2}[q(q-1)+q(p-q+1)-p-q+n]= \\
& =\frac{1}{2}(p q-p-q+n)=\frac{(p-1)(q-1)-1+n}{2} .
\end{aligned}
$$

Now we use Lemma 6.1 again and obtain that

$$
\tau\left(T_{q, p}\right)=\frac{(p-1)(q-1)-1+n}{2} .
$$

Using the lower bound of Equation (1) gives a different way to compute the slice genus of a torus link respect to what we did in [1]:

$$
g_{4}\left(T_{q, p}\right) \geqslant \frac{(p-1)(q-1)}{2}-\frac{1-n}{2}+1-n=\frac{(p-1)(q-1)+1-n}{2} .
$$

Since the Seifert algorithm applied to the standard diagram of $T_{q, p}$ gives the opposite inequality, we conclude that

$$
g_{4}\left(T_{q, p}\right)=\frac{(p-1)(q-1)+1-n}{2} \text { for any } q \leqslant p
$$

\subsection{Applications to Legendrian invariants}

We equip $S^{3}$ with its unique tight contact structure $\xi_{\text {st }}$, whose definition can be found in [2] Chapter 2. We want to prove that Equation (3) holds in this case:

$$
\operatorname{tb}(\mathcal{L})+|\operatorname{rot}(\mathcal{L})| \leqslant 2 \tau(L)-n .
$$

We remark that, if $\mathcal{D}=\mathcal{D}_{1} \cup \ldots \cup \mathcal{D}_{n}$ is a front projection of the Legendrian link $\mathcal{L}$ in the standard contact 3-sphere, the Thurston-Bennequin and rotation number of $\mathcal{L}$ are given by

$$
\operatorname{tb}(\mathcal{L})=\sum_{i=1}^{n} \operatorname{tb}_{i}(\mathcal{L}) \quad \text { and } \quad \operatorname{rot}(\mathcal{L})=\sum_{i=1}^{n} \operatorname{rot}_{i}(\mathcal{L})
$$

where

$$
\operatorname{tb}_{i}(\mathcal{L})=w\left(\mathcal{D}_{i}\right)+\operatorname{lk}\left(\mathcal{D}_{i}, \mathcal{D} \backslash \mathcal{D}_{i}\right)-\frac{1}{2} \# \mid \operatorname{cusps} \text { in } \mathcal{D}_{i} \mid
$$


and

$$
\operatorname{rot}_{i}(\mathcal{L})=\frac{1}{2}\left(\# \mid \text { downward cusps in } \mathcal{D}_{i}|-\#| \text { upward cusps in } \mathcal{D}_{i} \mid\right) ;
$$

here we denote with $w$ the writhe of a link diagram. We could simply say that

$$
\operatorname{tb}(\mathcal{L})=w(\mathcal{D})-\frac{1}{2} \# \mid \operatorname{cusps} \text { in } \mathcal{D} \mid
$$

and

$$
\operatorname{rot}(\mathcal{L})=\frac{1}{2}(\# \mid \text { downward cusps in } \mathcal{D}|-\#| \text { upward cusps in } \mathcal{D} \mid),
$$

but we need the previous definition in the following proof.

Proof of Proposition 1.5 If $\mathcal{L}$ is a Legendrian link then, from Chapter 12 in [7], we know that $\mathcal{L}$ can be represented by a grid diagram $D$ of the link $L^{*}$ (the mirror of $L$ ). This diagram $D$ is such that

$$
\begin{aligned}
& \frac{\mathrm{tb}_{i}(\mathcal{L})-\operatorname{rot}_{i}(\mathcal{L})+1}{2}=A_{i}\left(x^{+}\right) \quad \frac{\operatorname{tb}_{i}(\mathcal{L})+\operatorname{rot}_{i}(\mathcal{L})+1}{2}=A_{i}\left(x^{-}\right) \\
& \operatorname{tb}(\mathcal{L})-\operatorname{rot}(\mathcal{L})+1=M\left(x^{+}\right) \quad \operatorname{tb}(\mathcal{L})+\operatorname{rot}(\mathcal{L})+1=M\left(x^{-}\right),
\end{aligned}
$$

where $x^{ \pm}$are the grid states in $D$ obtained by taking a point in the northeast (southwest for $x^{-}$) corner of every square decorated with an $X \in \mathbb{X}$. Moreover, $A_{i}$ is defined as follows:

$$
A_{i}(x)=\mathcal{J}\left(x-\frac{1}{2}(\mathbb{X}+\mathbb{O}),\left(\mathbb{X}_{i}-\mathbb{O}_{i}\right)\right)-\frac{\operatorname{grd}(D)_{i}-1}{2} \quad \text { for any } x \in S(D)
$$

with $\mathbb{O}_{i} \subset \mathbb{O}$ and $\mathbb{X}_{i} \subset \mathbb{X}$ the markings on the $i$-th component of $L^{*}$ and $\operatorname{grd}(D)_{i}$ the number of elements in $\mathbb{O}_{i}$.

From Lemma 8.4.7 and Theorems 12.3.2 and 12.7.5 in [7] we have that $x^{ \pm}$represent non-torsion elements in the homology group $c G H^{-}\left(L^{*}\right)$; in fact these classes are the Legendrian grid invariants $\lambda^{ \pm}(\mathcal{L})$. Since $A(x)=\sum_{i=1}^{n} A_{i}(x)$ for every grid state $x$, we have that $M\left(x^{ \pm}\right)=2 A\left(x^{ \pm}\right)+1-n$. Therefore, Corollary 5.1 implies that $A\left(x^{ \pm}\right) \leqslant-\tau^{*}\left(L^{*}\right)$. Combining the latter claim with Corollary 3.5, that gives $\tau^{*}\left(L^{*}\right)=-\tau(L)$, we have

$$
\frac{\operatorname{tb}(\mathcal{L}) \mp \operatorname{rot}(\mathcal{L})+n}{2}=A\left(x^{ \pm}\right) \leqslant \tau(L)
$$

that is precisely Equation (3).

From Equation (3), together with Equation (1), we obtain the lower bound for the slice genus of Equation (4):

$$
\operatorname{tb}(\mathcal{L})+|\operatorname{rot}(\mathcal{L})| \leqslant 2 g_{4}(L)+n-2 .
$$

This bound is sharp for positive torus links, but here we show that there are other links for which this happens.

In Figure 17 we have a front projection $\mathcal{D}$ of a Legendrian two component link $\mathcal{L}$. The link type of $\mathcal{L}$ is the link $L 9_{19}^{n}$. A simple computation gives $\operatorname{tb}(\mathcal{L})=6$ and $\operatorname{rot}(\mathcal{L})=0$, therefore Equation (4) says that $g_{4}\left(L 9_{19}^{n}\right) \geqslant 3$. Since it is easy to see that the link represented by $\mathcal{D}$ can be unlinked by changing the four crossings highlighted in the picture, we have $g_{4}\left(L 9_{19}^{n}\right) \leqslant u\left(L 9_{19}^{n}\right)-1 \leqslant 3$ and then we conclude that $g_{4}\left(L 9_{19}^{n}\right)=3$. 


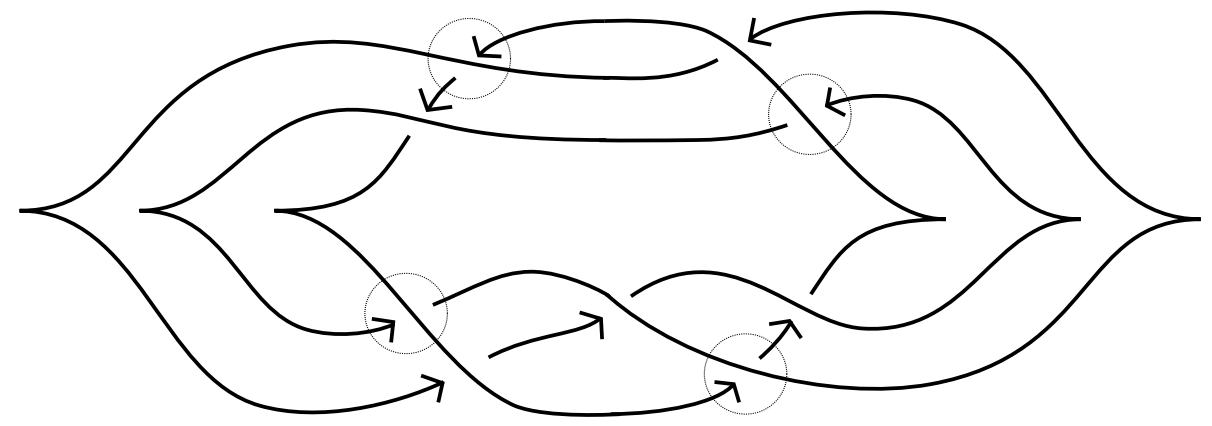

Figure 17: A diagram of the link $L 9_{19}^{n}$.

From Equation (3) we also have the upper bound for the maximal Thurston-Bennequin number of Proposition 1.6:

$$
\mathrm{TB}(L) \leqslant 2 \tau(L)-n
$$

and its refinement for quasi-alternating links given by Corollary 1.7. Although this bound is much less powerful than the Kauffmann or the HOMFLY polynomial, we can still get some interesting conclusions.

Consider a Legendrian link $\mathcal{L}$ such that each component $\mathcal{L}_{i}$ is algebraically unknotted. Then $\operatorname{tb}_{i}(\mathcal{L})=\operatorname{tb}\left(\mathcal{L}_{i}\right)$ and so $\operatorname{tb}(\mathcal{L})$ is precisely the sum of the Thurston-Bennequin numbers of its components. For example this happens for the Borromean link $B$, whose components $B_{i}$ are three (algebraically unknotted) unknots. It was shown in [6] that there is no Legendrian representation of $B$, where the Thurston-Bennequin number of each component is -1 ; in fact we have $\operatorname{TB}(B)=-4$, while $\operatorname{TB}(\bigcirc)=-1$. In particular, this means that the difference between $\operatorname{TB}(B)$ and the sum of $\operatorname{TB}\left(B_{i}\right)$ is -1 .

We prove Proposition 1.8, where we give a family of two components links $L^{k}$ such that the components of $L^{k}$ are two unknots with $\operatorname{lk}\left(L_{1}^{k}, L_{2}^{k}\right)=0$ and the difference between $\operatorname{TB}\left(L^{k}\right)$ and the sum of $\operatorname{TB}\left(L_{i}^{k}\right)$ is actually arbitrarily small, improving the latter result for $B$. The links $L^{k}$ are shown in Figure 1.

Proof of Proposition 1.8 Since for every $k \geqslant 0$ the link $L^{k}$ is non-split alternating, we can easily compute the signature that is equal to $3+2 k$. Now we apply Corollary 1.7 and we obtain that $\operatorname{TB}\left(L^{k}\right) \leqslant-4-2 k$.

\section{References}

[1] A. Cavallo, On the slice genus and some concordance invariants of links, J. Knot Theory Ramifications, 24 (2015), no. 4, 1550021.

[2] H. Geiges, An introduction to contact topology, Cambridge University Press, volume 109 of Cambridge Studies in Advanced Mathematics, 2008.

[3] J. Hom and Z. Wu, Four-ball genus bounds and a refinement of the Ozsváth-Szabó $\tau$-invariant, J. Symplectic Geom., 14 (2016), no. 1, pp. 305-323.

[4] A. Kawauchi, T. Shibuya and S. Suzuki, Descriptions on surfaces in four-space. I. Normal forms, Math. Sem. Notes Kobe Univ., 10 (1982), no. 1, pp. 75-125. 
[5] C. Manolescu, P. Ozsváth, Z. Szabó and D. Thurston, On combinatorial link Floer homology, Geom. Topol., 11 (2007), pp. 2339-2412.

[6] K. Mohnke, Legendrian links of topological unknots, AMS, volume 279 of Contemporary Mathematics, 2001, pp. 209-211.

[7] P. Ozsváth, A. Stipsicz and Z. Szabó, Grid homology for knots and links, AMS, volume 208 of Mathematical Surveys and Monographs, 2015.

[8] P. Ozsváth and Z. Szabó, Holomorphic disks and knot invariants, Adv. Math., 186 (2004), no. 1, pp. 58-116.

[9] P. Ozsváth and Z. Szabó, On the Heegaard Floer homology of branched double-covers, Adv. Math., 194 (2005), no. 1, pp. 1-33.

[10] J. Pardon, The link concordance invariant from Lee homology, Algebr. Geom. Topol., 12 (2012), no. 2, pp. 1081-1098.

[11] O. Plamenevskaya, Bounds for the Thurston-Bennequin number from Floer homology, Algebr. Geom. Topol., 4 (2004), pp. 399-406.

[12] J. Rasmussen, Floer homology and knot complements, PhD thesis, Harvard University, 2003.

[13] L. Rudolph, An obstruction to sliceness via contact geometry and "classical" gauge theory, Invent. Math., 119 (1995), no. 1, pp. 155-163.

[14] S. Sarkar, Grid diagrams and the Ozsváth-Szabó tau-invariant, Math. Res. Let., 18 (2011), no. 6, pp. 1239-1257.

Alfréd Rényi Institute of Mathematics, Budapest 1053, Hungary

cavallo_alberto@phd.ceu.edu 